Research Article

\title{
Pure Traveling Wave Solutions for Three Nonlinear Fractional Models
}

\author{
Qinjun Li $\mathbb{D}^{1},{ }^{1}$ Danyal Soybaş $\mathbb{D}^{2},{ }^{2}$ Onur Alp Ilhan $\mathbb{D}^{2},{ }^{2}$ Gurpreet Singh $\mathbb{D}^{1}{ }^{3}$ \\ and Jalil Manafian $\mathbb{i D}^{4}$ \\ ${ }^{1}$ Department of Ecological Engineering, Xining Urban Vocational \& Technical College, Xining City, Qinghai Province, China \\ ${ }^{2}$ Department of Mathematics, Faculty of Education, Erciyes University, 38039 Melikgazi, Kayseri, Turkey \\ ${ }^{3}$ Department of Mathematics, Sant Baba Bhag Singh University, 144030, Jalandhar, India \\ ${ }^{4}$ Department of Applied Mathematics, Faculty of Mathematical Sciences, University of Tabriz, Tabriz, Iran
}

Correspondence should be addressed to Jalil Manafian; j_manafianheris@tabrizu.ac.ir

Received 28 December 2020; Revised 25 January 2021; Accepted 16 March 2021; Published 10 April 2021

Academic Editor: Manuel De León

Copyright ( 2021 Qinjun Li et al. This is an open access article distributed under the Creative Commons Attribution License, which permits unrestricted use, distribution, and reproduction in any medium, provided the original work is properly cited.

Three nonlinear fractional models, videlicet, the space-time fractional $(1+1)$ Boussinesq equation, $(2+1)$-dimensional breaking soliton equations, and SRLW equation, are the important mathematical approaches to elucidate the gravitational water wave mechanics, the fractional quantum mechanics, the theoretical Huygens' principle, the movement of turbulent flows, the ion osculate waves in plasma physics, the wave of leading fluid flow, etc. This paper is devoted to studying the dynamics of the traveling wave with fractional conformable nonlinear evaluation equations (NLEEs) arising in nonlinear wave mechanics. By utilizing the oncoming $\exp (-\Theta(q))$-expansion technique, a series of novel exact solutions in terms of rational, periodic, and hyperbolic functions for the fractional cases are derived. These types of long-wave propagation phenomena played a dynamic role to interpret the water waves as well as mathematical physics. Here, the form of the accomplished solutions containing the hyperbolic, rational, and trigonometric functions is obtained. It is demonstrated that our proposed method is further efficient, general, succinct, powerful, and straightforward and can be asserted to install the new exact solutions of different kinds of fractional equations in engineering and nonlinear dynamics.

\section{Introduction}

The solutions of fractional partial differential equations (FPDEs) are often of interest and are applied in practical life. One of the main purposes of mathematical physics is to determine the exact solutions. Some scholars had perused the plenty of physical wave equations. Therefore, several analytical methods were systematically developed and applied to achieve exact and approximate solutions of fractional ordinary and partial differential equations with applications in various fields of sciences like fluid flow, mechanics, biology, nonlinear optics, substance energy, system identification, and geooptical filaments which are expressed in fractional forms [1-11].

In the past few decades, a lot of studies have been executed to find the new and further exact traveling wave solu- tion of space-time fractional PDEs by many research. With the collaboration of potential symbolic computer programming software, they have been appointed for researching appropriate solution to the nonlinear space-time fractional PDEs by executing powerful techniques, for example, the $\tan -(\phi / 2)$ expansion method, the sin-Gordon expansion method, the $G^{\prime} / G$ expansion method, and the advanced exponential expansion method [12-25].

This work mainly investigates three nonlinear fractional models by utilizing the oncoming $\exp (-\Theta(q))$-expansion method [17, 25]. Recently, Bashar and Roshid [17] and Rahhman et al. [25] have studied this technique to some fractional and nonfractional NLEEs. They found that this introduced method provides some simple general form of traveling wave solutions. Rahhman et al. [25] did not give 
any fruitful discussion about fractional NLEEs with this proposed method. The important idea of this method is too explicit: the exact solutions of NLEEs satisfy the nonlinear ODE, $\Theta^{\prime}(q)+\theta \exp (\Theta(q))+\delta \exp (-\Theta(q))$, where $\theta$ and $\delta$ are real parameters.

The space-time fractional Boussinesq equation with the $\rho$-derivative $[26,27]$ is presented as follows:

$$
D_{t}^{2 \alpha} \Psi+b D_{x}^{2 \alpha} \Psi+\beta D_{x}^{2 \alpha}\left(\Psi^{2}\right)+\gamma D_{x}^{4 \alpha} \Psi=0, \quad t>0,0<\alpha \leq 1,
$$

where $\Psi(x, t)$ is the vertical deflection. In Ref. [28], authors constructed an analytical solution for both linear and nonlinear time-fractional Boussinesq equations by an iterative method. Also, Hemeda [29] studied the fractional Boussinesq-like equation via a new iterative method. Authors of [30] investigated nonlinear two-point boundary value problem to the fractional Boussinesq-like equation. Furthermore, the space-time fractional breaking soliton equations $[27,31]$ are taken in the following form:

$$
\begin{gathered}
D_{t}^{2 \alpha} \Psi+a D_{x^{2} y}^{3 \alpha} \Psi+4 a \Psi\left(D_{x}^{\alpha} \Omega\right)+4 a \Omega\left(D_{x}^{\alpha} \Psi\right)=0, \quad t>0,0<\alpha \leq 1, \\
D_{y}^{\alpha} \Psi-D_{x}^{\alpha} \Omega=0 .
\end{gathered}
$$

Meng and Feng [32] used an auxiliary equation method to the space-time fractional $(2+1)$-dimensional breaking soliton equation. Authors of $[27,33,34]$ discussed the spacetime fractional SRLW equation (STFSRLWE) in the following case:

$$
\begin{gathered}
D_{t}^{2 \alpha} \Psi+D_{x}^{2 \alpha} \Psi+\Psi D_{t}^{\alpha}\left(D_{x}^{\alpha} \Psi\right)+D_{t}^{\alpha} \Psi D_{x}^{\alpha} \Psi \\
+D_{t}^{2 \alpha}\left(D_{x}^{2 \alpha} \Psi\right)=0, \quad t>0,0<\alpha \leq 1
\end{gathered}
$$

The functional variable method, exp-function method, and $\left(G^{\prime} / G\right)$-expansion method to the fractional SRLW equation in the sense of the modified Riemann-Liouville derivative were utilized in Ref. [35]. The interested readers can see more works in Refs. ([36-50]). Inc and coworkers presented the new soliton structures to some time-fractional nonlinear differential equations with a conformable derivative via the Ricatti-Bernoulli sub-ODE method [51]. Salahshour and colleagues worked on the truncated $M$ -fractional derivative as a novel and effective derivative under interval uncertainty and investigated the existence and uniqueness conditions of the solution [52]. Authors of [53] studied a coupled nonlinear Maccari's system which describes the motion of isolated waves localized in a small part of space. Authors of [54] employed the exponential function method for the combined $\mathrm{KdV}-\mathrm{mKdV}$ equation.

One technique, videlicet, the oncoming $\exp (-\Theta(q))$ -expansion technique, was employed by many researchers for solving the number of nonlinear PDEs or fractional PDEs. For both methods, the interested readers can refer for the first technique to Refs. ([55-59]).
The pattern of this article is summarized as follows. In Sections 2 and 3, the properties and the detail of technique are given, which are to be utilized for getting the exact solutions of the fractional Boussinesq, breaking soliton, and SRLW equations along with numerical simulation and details of graph in Sections 4-7. Finally, some conclusions are given in the end.

\section{Analysis of $\mu$-Derivative}

Definition 1 (see [60]). Definition of $\mu$-derivative: let $\lambda$ : [0; $1) \rightarrow \mathbf{R}$; then, the $\mu$-derivative of $\lambda$ of order $\mu$ is defined as

$$
D_{t}^{\mu}(\lambda)(t)=\lim _{\varepsilon \rightarrow 0} \frac{\lambda\left[t+\varepsilon(t+(1 / \Gamma(\mu)))^{1-\mu}\right]-\lambda(t)}{\varepsilon}, \quad \mu \in(0,1], t>0 .
$$

The features and novel theorems will be utilized as follows:The proofs of the above $\mu$-derivative properties are obviously given in [60].

Theorem 2. Let $\mu \in(0,1] ; \lambda, \vartheta$ be $\mu$-differentiable at point $t$; therefore, we get

$$
\text { (1) } \begin{aligned}
D_{t}^{\mu}(a \lambda(t)+b \vartheta(t))=a D_{t}^{\alpha}(\lambda(t))+b D_{t}^{\alpha}(\vartheta(t)) \text {, for } a, b \\
\in \mathbf{R}
\end{aligned}
$$

(2) $D_{t}^{\mu}(c)=0$, for $c \in \mathbf{R}$

(3) $D_{t}^{\mu}(\lambda(t) \vartheta(t))=\lambda(t) D_{t}^{\mu}(\vartheta(t))+\vartheta(t) D_{t}^{\mu}(\lambda(t))$

(4) $D_{t}^{\mu}(\lambda(t) / \vartheta(t))=\left(\lambda(t) D_{t}^{\alpha}(\vartheta(t))-\vartheta(t) D_{t}^{\mu}(\lambda(t))\right) / \vartheta^{2}(t)$

(5) $D_{t}^{\mu} \lambda(t)=(t+(1 / \Gamma(\mu)))^{1-\mu} d \lambda(t) / d t$

We have the following features as follows:

$$
\begin{aligned}
D_{t}^{\mu} \sin (t) & =\lim _{\varepsilon \rightarrow 0} \frac{\sin \left[t+\varepsilon(t+(1 / \Gamma(\mu)))^{1-\mu}\right]-\sin (t)}{\varepsilon} \\
& =\left(t+\frac{1}{\Gamma(\mu)}\right)^{1-\alpha} \cos (t), \\
D_{t}^{\mu} \cos (t) & =\lim _{\varepsilon \rightarrow 0} \frac{\cos \left[t+\varepsilon(t+(1 / \Gamma(\mu)))^{1-\mu}\right]-\cos (t)}{\varepsilon} \\
& =-\left(t+\frac{1}{\Gamma(\mu)}\right)^{1-\mu} \sin (t), \\
D_{t}^{\mu} \exp (t) & =\lim _{\varepsilon \rightarrow 0} \frac{\exp \left[t+\varepsilon(t+(1 / \Gamma(\mu)))^{1-\mu}\right]-\exp (t)}{\varepsilon} \\
& =\left(t+\frac{1}{\Gamma(\mu)}\right)^{1-\alpha} \exp (t) .
\end{aligned}
$$

Theorem 3. Let $\lambda:[0 ; 1) \rightarrow \boldsymbol{R}$; be a function such that $\lambda$ is differentiable and also $\mu$-differentiable. Also, let $\lambda$ be a 
differentiable function defined in the range of $\vartheta$. Then, we get

$$
D_{t}^{\mu}(\lambda \circ \vartheta)(t)=\left(t+\frac{1}{\Gamma(\mu)}\right)^{1-\mu} \vartheta^{\prime}(t) \lambda^{\prime}(\vartheta(t))
$$

where prime denotes the classical derivatives with respect to $t$.

\section{The Oncoming $\exp (-\Theta(q))$-Expansion Technique}

This method was summarized and improved for achieving the analytic solutions of NLPDEs.

Step 1. Assume that a nonlinear partial differential equation is given in the general form as follows:

$$
\mathscr{F}\left(u, u_{x}, u_{y}, u_{t}, u_{x x}, u_{t t}, \cdots\right)=0 .
$$

After simple algebraic operations, this equation is transformed into an ordinary differential equation (ODE) with the below transformation:

$$
u(x, y, t)=U(q), q=x+k y+\omega t
$$

as well as into nonlinear ODE:

$$
\delta\left(U, U^{\prime}, \omega U^{\prime}, k U^{\prime}, U^{\prime \prime}, \omega^{2} U^{\prime \prime}, \cdots\right)=0 .
$$

Step 2. Then, assume that the searched wave solutions of equation (9) have the following representation:

$$
U(q)=\sum_{j=0}^{\chi} \pi_{j} Y^{j}(q)+\sum_{j=0}^{\chi} \tau_{j} Y^{-j}(q)
$$

where $Y(q)=\exp (-\Theta(q))$ and $\pi_{j}(0 \leq j \leq \chi), \tau_{j}(0 \leq j \leq \chi)$ are constants to be determined, such that $\pi_{\chi}, \tau_{\chi} \neq 0$, and $\Theta=\Theta$ $(q)$ is the solution of the following first-order differential equation:

$$
\Theta^{\prime}=\delta Y^{-1}(q)+Y(q)+\theta .
$$

If we try to find the solution of (11), then we obtain special solutions that vary according to the state of the coefficients:

Solution 1 (hyperbolic function solution). If $\delta \neq 0$ and $\theta^{2}-4 \delta>0$, then we achieve

$$
\Theta(q)=\ln \left(-\frac{\sqrt{\theta^{2}-4 \delta}}{2 \delta} \tanh \left(\frac{\sqrt{\theta^{2}-4 \delta}}{2}(q+\Sigma)\right)-\frac{\theta}{2 \delta}\right)
$$

where $\Sigma$ is the integral constant.
Solution 2 (trigonometric function solution). If $\delta \neq 0$ and $\theta^{2}-4 \delta<0$, afterward we achieve

$$
\Theta(q)=\ln \left(\frac{\sqrt{-\theta^{2}+4 \delta}}{2 \delta} \tan \left(\frac{\sqrt{-\theta^{2}+4 \delta}}{2}(q+\Sigma)\right)-\frac{\theta}{2 \delta}\right) .
$$

Solution 3. If $\delta=0, \theta \neq 0$, and $\theta^{2}-4 \delta>0$, afterward we achieve

$$
\Theta(q)=-\ln \left(\frac{\theta}{\exp (\theta(q+\Sigma))-1}\right)
$$

Solution 4. If $\delta \neq 0, \theta \neq 0$, and $\theta^{2}-4 \delta=0$, afterward we achieve

$$
\Theta(q)=\ln \left(-\frac{2 \theta(q+\Sigma)+4}{\theta^{2}(q+\Sigma)}\right) .
$$

Solution 5. If $\delta=0, \theta=0$, and $\theta^{2}-4 \delta=0$, afterward we achieve

$$
\Theta(q)=\ln (q+\Sigma)
$$

where $\pi_{j}(0 \leq j \leq \chi), \tau_{j}(0 \leq j \leq \chi), \theta$, and $\delta$ are also the constants to be explored later. As usual, for determining $\chi$, the highest-order derivative should be balanced with the highest-order nonlinear terms in equation (10). However, the positive integer $\chi$ can be determined in this way.

Step 3. Following these operations, according to the $m$ value obtained above, let (11) be substituted into equation (10). Therefore, we obtain a set of algebraic equations that contains $Y^{s}(q)(s=0,1,2, \cdots)$. Then, setting each coefficient of $\exp (-\Theta(q) s)$ to zero, we can get a set of overdetermined equations for $\pi_{0}, \pi_{1}, \tau_{1}, \cdots, \pi_{\chi}, \tau_{\chi}, \theta$, and $\delta$. Since the obtained algebraic equation system will be difficult to solve manually, symbolic computation such as Maple can be used at this stage. Assume that the estimation of the constants can be gotten by fathoming the mathematical conditions in step 2. Substituting the estimations of the constants together with the arrangements of equation (11), we will acquire new and far reaching precise traveling wave arrangements of the nonlinear development equation (7).

The $\Sigma$ has the following features as follows:

$$
\begin{gathered}
U(q) \simeq \xi Y^{\chi}, \\
U^{\prime}(q) \simeq \xi Y^{\chi-1} Y^{\prime}=\xi Y^{\chi+1}, \\
\left.U^{\prime \prime}(q)\right) \simeq \xi Y^{\chi+2}, \\
(U(q))^{2} \simeq \xi^{2} Y^{2 \chi},
\end{gathered}
$$


where $\xi=\pi_{\chi}$. Balancing $U^{\prime \prime}$ with $U^{2}$ yields

$$
\chi+2=2 \chi \Rightarrow \chi=2 .
$$

\section{The First Equation}

By utilizing the following transformation:

$$
q=k\left(x+\frac{1}{\Gamma(\mu)}\right)^{\mu}-\omega\left(t+\frac{1}{\Gamma(\mu)}\right)^{\mu},
$$

then, equation (1) transformed to

$$
\omega^{2} \Psi^{\prime \prime}+b k^{2} \Psi^{\prime \prime}+\beta k^{2}\left(\Psi^{2}\right)^{\prime \prime}+\gamma k^{4} \Psi^{\prime \prime \prime \prime}=0,
$$

where $\Psi^{\prime}=d \Psi / d q$, and by integrating equation (20) twice with respect to $q$, it can be seen as

$$
\left(\omega^{2}+b k^{2}\right) \Psi+\beta k^{2} \Psi^{2}+\gamma k^{4} \Psi^{\prime \prime}=0 .
$$

The balance number will be obtained $\chi=2$ by using the balance principle. Then, the exact solution is given as

$$
\Psi(q)=\pi_{0}+\pi_{1} Y(q)+\pi_{2} Y^{2}(q)+\frac{\tau_{1}}{Y(q)}+\frac{\tau_{2}}{Y^{2}(q)} .
$$

Firstly, we substitute the expressions of $\Psi(q)$ in (22) into (21) and collect all terms with the same order of $Y(q)$. Then, by equating the coefficient of each polynomial to zero, we obtain a set of algebraic equations as follows:

$$
\left\{\begin{array}{l}
6 \gamma k^{4} \tau_{2}+\beta k^{2} \tau_{2}^{2}=0 \\
10 \gamma k^{4} \theta \tau_{2}+2 \gamma k^{4} \tau_{1}+2 \beta k^{2} \tau_{1} \tau_{2}=0 \\
4 \gamma k^{4} \theta^{2} \tau_{2}+8 \delta \gamma k^{4} \tau_{2}+3 \gamma k^{4} \theta \tau_{1}+2 \beta k^{2} \pi_{0} \tau_{2}+\beta k^{2} \tau_{1}^{2}+b k^{2} \tau_{2}+\omega^{2} \tau_{2}=0 \\
6 \delta \gamma k^{4} \theta \tau_{2}+\gamma k^{4} \theta^{2} \tau_{1}+2 \delta \gamma k^{4} \tau_{1}+2 \beta k^{2} \pi_{0} \tau_{1}+2 \beta k^{2} \pi_{1} \tau_{2}+b k^{2} \tau_{1}+\omega^{2} \tau_{1}=0, \\
2 \delta^{2} \gamma k^{4} \tau_{2}+\delta \gamma k^{4} \theta \tau_{1}+\gamma k^{4} \theta \pi_{1}+2 \gamma k^{4} \pi_{2}+\beta k^{2} \pi_{0}^{2}+2 \beta k^{2} \pi_{1} \tau_{1}+2 \beta k^{2} \pi_{2} \tau_{2}+b k^{2} \pi_{0}+\omega^{2} \pi_{0}=0 \\
\gamma k^{4} \theta^{2} \pi_{1}+2 \delta \gamma k^{4} \pi_{1}+6 \gamma k^{4} \theta \pi_{2}+2 \beta k^{2} \pi_{0} \pi_{1}+2 \beta k^{2} \pi_{2} \tau_{1}+b k^{2} \pi_{1}+\omega^{2} \pi_{1}=0, \\
3 \delta \gamma k^{4} \theta \pi_{1}+4 \gamma k^{4} \theta^{2} \pi_{2}+8 \delta \gamma k^{4} \pi_{2}+2 \beta k^{2} \pi_{0} \pi_{2}+\beta k^{2} \pi_{1}^{2}+b k^{2} \pi_{2}+\omega^{2} \pi_{2}=0, \\
2 \delta^{2} \gamma k^{4} \pi_{1}+10 \delta \gamma k^{4} \theta \pi_{2}+2 \beta k^{2} \pi_{1} \pi_{2}=0 \\
6 \delta^{2} \gamma k^{4} \pi_{2}+\beta k^{2} \pi_{2}^{2}=0 .
\end{array}\right.
$$

First:

$$
\begin{aligned}
\omega & =k \sqrt{\gamma k^{2} \theta^{2}-4 \delta \gamma k^{2}-b}, \pi_{0} \\
& =-\frac{k^{2} \gamma\left(\theta^{2}+2 \delta\right)}{\beta}, \tau_{1} \\
& =-6 \frac{k^{2} \gamma \theta}{\beta}, \tau_{2}=-6 \frac{k^{2} \gamma}{\beta} .
\end{aligned}
$$

According to Family I, (22) becomes

$$
\begin{aligned}
\Psi_{1}(q)= & -\frac{k^{2} \gamma\left(\theta^{2}+2 \delta\right)}{\beta} \\
& -\frac{6\left(k^{2} \gamma \theta / \beta\right)}{\left(-\left(\sqrt{\theta^{2}-4 \delta} / 2 \delta\right) \tanh \left(\left(\sqrt{\theta^{2}-4 \delta} / 2\right)(q+\Sigma)\right)-(\theta / 2 \delta)\right)} \\
& -\frac{6\left(k^{2} \gamma / \beta\right)}{\left(-\left(\sqrt{\theta^{2}-4 \delta} / 2 \delta\right) \tanh \left(\left(\sqrt{\theta^{2}-4 \delta} / 2\right)(q+\Sigma)\right)-(\theta / 2 \delta)\right)^{2}},
\end{aligned}
$$

where

$$
\begin{aligned}
q= & k\left(x+\frac{1}{\Gamma(\mu)}\right)^{\mu} \\
& -k \sqrt{\gamma k^{2} \theta^{2}-4 \delta \gamma k^{2}-b}\left(t+\frac{1}{\Gamma(\mu)}\right)^{\mu} .
\end{aligned}
$$

According to Family II, (22) becomes

$$
\begin{aligned}
\Psi_{2}(q) & =-\frac{k^{2} \gamma\left(\theta^{2}+2 \delta\right)}{\beta} \\
& -\frac{6\left(k^{2} \gamma \theta / \beta\right)}{\left(\left(\sqrt{-\theta^{2}+4 \delta} / 2 \delta\right) \tan \left(\left(\sqrt{-\theta^{2}+4 \delta} / 2\right)(q+\Sigma)\right)-(\theta / 2 \delta)\right)} \\
& -\frac{6\left(k^{2} \gamma / \beta\right)}{\left(\left(\sqrt{-\theta^{2}+4 \delta} / 2 \delta\right) \tan \left(\left(\sqrt{-\theta^{2}+4 \delta} / 2\right)(q+\Sigma)\right)-(\theta / 2 \delta)\right)^{2}},
\end{aligned}
$$


where

$$
q=k\left(x+\frac{1}{\Gamma(\mu)}\right)^{\mu}-k \sqrt{\gamma k^{2} \theta^{2}-4 \delta \gamma k^{2}-b}\left(t+\frac{1}{\Gamma(\mu)}\right)^{\mu} .
$$

According to Family III, (22) can be written as

$$
\begin{aligned}
\Psi_{3}(q)= & -\frac{k^{2} \gamma \theta^{2}}{\beta}-6 \frac{k^{2} \gamma \theta}{\beta}\left(\frac{\theta}{\exp (\theta(q+\Sigma))-1}\right) \\
& -6 \frac{k^{2} \gamma}{\beta}\left(\frac{\theta}{\exp (\theta(q+\Sigma))-1}\right)^{2},
\end{aligned}
$$

where

$$
q=k\left(x+\frac{1}{\Gamma(\mu)}\right)^{\mu}-k \sqrt{\gamma k^{2} \theta^{2}-b}\left(t+\frac{1}{\Gamma(\mu)}\right)^{\mu} .
$$

According to Family IV, (22) becomes

$$
\begin{aligned}
\Psi_{4}(q)= & -\frac{k^{2} \gamma\left(\theta^{2}+2 \delta\right)}{\beta}-6 \frac{k^{2} \gamma \theta}{\beta}\left(-\frac{\theta^{2}(q+E)}{2 \theta(q+\Sigma)+4}\right) \\
& -6 \frac{k^{2} \gamma}{\beta}\left(-\frac{\theta^{2}(q+E)}{2 \theta(q+\Sigma)+4}\right)^{2},
\end{aligned}
$$

where

$$
q=k\left(x+\frac{1}{\Gamma(\mu)}\right)^{\mu}-k \sqrt{-b}\left(t+\frac{1}{\Gamma(\mu)}\right)^{\mu} .
$$

Second:

$$
\begin{aligned}
\omega & =k \sqrt{-\gamma k^{2} \theta^{2}+4 \delta \gamma k^{2}-b}, \pi_{0}=-\frac{6 k^{2} \gamma \delta}{\beta}, \tau_{1} \\
& =-\frac{6 k^{2} \gamma \theta}{\beta}, \tau_{2}=-\frac{6 k^{2} \gamma}{\beta} .
\end{aligned}
$$

According to Family I, (22) can be written as

$$
\begin{aligned}
\Psi_{5}(q) & =-\frac{6 k^{2} \gamma \delta}{\beta} \\
& -\frac{6\left(k^{2} \gamma \theta / \beta\right)}{\left(-\left(\sqrt{\theta^{2}-4 \delta} / 2 \delta\right) \tanh \left(\left(\sqrt{\theta^{2}-4 \delta} / 2\right)(q+\Sigma)\right)-(\theta / 2 \delta)\right)} \\
& -\frac{6\left(k^{2} \gamma / \beta\right)}{\left(-\left(\sqrt{\theta^{2}-4 \delta} / 2 \delta\right) \tanh \left(\left(\sqrt{\theta^{2}-4 \delta} / 2\right)(q+\Sigma)\right)-(\theta / 2 \delta)\right)^{2}},
\end{aligned}
$$

where

$$
q=k\left(x+\frac{1}{\Gamma(\mu)}\right)^{\mu}-k \sqrt{-\gamma k^{2} \theta^{2}+4 \delta \gamma k^{2}-b}\left(t+\frac{1}{\Gamma(\mu)}\right)^{\mu} .
$$

According to Family II, (22) can be written as

$$
\begin{aligned}
\Psi_{6}(q) & =-\frac{6 k^{2} \gamma \delta}{\beta} \\
& -\frac{6\left(k^{2} \gamma \theta / \beta\right)}{\left(\left(\sqrt{-\theta^{2}+4 \delta} / 2 \delta\right) \tan \left(\left(\sqrt{-\theta^{2}+4 \delta} / 2\right)(q+\Sigma)\right)-(\theta / 2 \delta)\right)} \\
& -\frac{6\left(k^{2} \gamma / \beta\right)}{\left(\left(\sqrt{\theta^{2}-4 \delta} / 2 \delta\right) \tan \left(\left(\sqrt{-\theta^{2}+4 \delta} / 2\right)(q+\Sigma)\right)-(\theta / 2 \delta)\right)^{2}},
\end{aligned}
$$

where

$$
q=k\left(x+\frac{1}{\Gamma(\mu)}\right)^{\mu}-k \sqrt{-\gamma k^{2} \theta^{2}+4 \delta \gamma k^{2}-b}\left(t+\frac{1}{\Gamma(\mu)}\right)^{\mu} .
$$

According to Family III, (22) can be written as

$$
\begin{aligned}
\Psi_{7}(q)= & -6 \frac{k^{2} \gamma \theta}{\beta}\left(\frac{\theta}{\exp (\theta(q+\Sigma))-1}\right) \\
& -6 \frac{k^{2} \gamma}{\beta}\left(\frac{\theta}{\exp (\theta(q+\Sigma))-1}\right)^{2},
\end{aligned}
$$

where

$$
q=k\left(x+\frac{1}{\Gamma(\mu)}\right)^{\mu}-k \sqrt{-\gamma k^{2} \theta^{2}-b}\left(t+\frac{1}{\Gamma(\mu)}\right)^{\mu} .
$$

According to Family IV, (22) becomes

$$
\begin{aligned}
\Psi_{8}(q)= & -\frac{6 k^{2} \gamma \delta}{\beta}-6 \frac{k^{2} \gamma \theta}{\beta}\left(-\frac{\theta^{2}(q+\Sigma)}{2 \theta(q+E)+4}\right) \\
& -6 \frac{k^{2} \gamma}{\beta}\left(-\frac{\theta^{2}(q+\Sigma)}{2 \theta(q+\Sigma)+4}\right)^{2},
\end{aligned}
$$

where

$$
q=k\left(x+\frac{1}{\Gamma(\mu)}\right)^{\mu}-k \sqrt{-b}\left(t+\frac{1}{\Gamma(\mu)}\right)^{\mu} .
$$

Third:

$$
\begin{aligned}
\omega & =k \sqrt{\gamma k^{2} \theta^{2}-4 \delta \gamma k^{2}-b}, \pi_{0}=-\frac{k^{2} \gamma\left(\theta^{2}+2 \delta\right)}{\beta}, \pi_{1} \\
& =-6 \frac{k^{2} \gamma \theta}{\beta}, \pi_{2}=-6 \frac{k^{2} \gamma}{\beta} .
\end{aligned}
$$


According to Family I, (22) becomes

$$
\begin{aligned}
\Psi_{9}(q)= & -\frac{k^{2} \gamma\left(\theta^{2}+2 \delta\right)}{\beta}-\frac{6 k^{2} \gamma \theta}{\beta} \\
& \cdot\left(-\frac{\sqrt{\theta^{2}-4 \delta}}{2 \delta} \tanh \left(\frac{\sqrt{\theta^{2}-4 \delta}}{2}(q+\Sigma)\right)-\frac{\theta}{2 \delta}\right) \\
& -6 \frac{k^{2} \gamma}{\beta}\left(-\frac{\sqrt{\theta^{2}-4 \delta}}{2 \delta} \tanh \left(\frac{\sqrt{\theta^{2}-4 \delta}}{2}(q+\Sigma)\right)-\frac{\theta}{2 \delta}\right)^{2},
\end{aligned}
$$

where

$$
q=k\left(x+\frac{1}{\Gamma(\mu)}\right)^{\mu}-k \sqrt{\gamma k^{2} \theta^{2}-4 \delta \gamma k^{2}-b}\left(t+\frac{1}{\Gamma(\mu)}\right)^{\mu} .
$$

According to Family II, (22) can be written as

$$
\begin{aligned}
\Psi_{10}(q)= & -\frac{k^{2} \gamma\left(\theta^{2}+2 \delta\right)}{\beta}-\frac{6 k^{2} \gamma \theta}{\beta} \\
& \cdot\left(\frac{\sqrt{-\theta^{2}+4 \delta}}{2 \delta} \tan \left(\frac{\sqrt{-\theta^{2}+4 \delta}}{2}(q+\Sigma)\right)-\frac{\theta}{2 \delta}\right) \\
& -6 \frac{k^{2} \gamma}{\beta}\left(\frac{\sqrt{-\theta^{2}+4 \delta}}{2 \delta} \tan \left(\frac{\sqrt{-\theta^{2}+4 \delta}}{2}(q+\Sigma)\right)-\frac{\theta}{2 \delta}\right)^{2},
\end{aligned}
$$

where

$$
q=k\left(x+\frac{1}{\Gamma(\mu)}\right)^{\mu}-k \sqrt{\gamma k^{2} \theta^{2}-4 \delta \gamma k^{2}-b}\left(t+\frac{1}{\Gamma(\mu)}\right)^{\mu} .
$$

According to Family III, (22) can be written as

$$
\begin{aligned}
\Psi_{11}(q)= & -\frac{k^{2} \gamma \theta^{2}}{\beta}-6 \frac{k^{2} \gamma \theta}{\beta}\left(\frac{\exp (\theta(q+\Sigma))-1}{\theta}\right) \\
& -6 \frac{k^{2} \gamma}{\beta}\left(\frac{\exp (\theta(q+\Sigma))-1}{\theta}\right)^{2},
\end{aligned}
$$

where

$$
q=k\left(x+\frac{1}{\Gamma(\alpha)}\right)^{\alpha}-k \sqrt{\gamma k^{2} \theta^{2}-b}\left(t+\frac{1}{\Gamma(\alpha)}\right)^{\alpha}
$$

According to Family IV, (22) can be written as

$$
\begin{aligned}
\Psi_{12}(q)= & -\frac{k^{2} \gamma\left(\theta^{2}+2 \delta\right)}{\beta}-6 \frac{k^{2} \gamma \theta}{\beta}\left(-\frac{2 \theta(q+\Sigma)+4}{\theta^{2}(q+E)}\right) \\
& -6 \frac{k^{2} \gamma}{\beta}\left(-\frac{2 \theta(q+\Sigma)+4}{\theta^{2}(q+E)}\right)^{2},
\end{aligned}
$$

where

$$
q=k\left(x+\frac{1}{\Gamma(\mu)}\right)^{\mu}-k \sqrt{-b}\left(t+\frac{1}{\Gamma(\mu)}\right)^{\mu}
$$

Fourth:

$$
\begin{aligned}
\omega & =k \sqrt{-\gamma k^{2} \theta^{2}+4 \delta \gamma k^{2}-b}, \pi_{0}=-\frac{6 k^{2} \gamma \delta}{\beta}, \pi_{1} \\
& =-\frac{6 k^{2} \gamma \theta}{\beta}, \pi_{2}=-\frac{6 k^{2} \gamma}{\beta} .
\end{aligned}
$$

According to Family I, (22) becomes

$$
\begin{aligned}
\Psi_{13}(q)= & -\frac{6 k^{2} \gamma \delta}{\beta} \\
& -6 \frac{k^{2} \gamma \theta}{\beta}\left(-\frac{\sqrt{\theta^{2}-4 \delta}}{2 \delta} \tanh \left(\frac{\sqrt{\theta^{2}-4 \delta}}{2}(q+\Sigma)\right)-\frac{\theta}{2 \delta}\right) \\
& -6 \frac{k^{2} \gamma}{\beta}\left(-\frac{\sqrt{\theta^{2}-4 \delta}}{2 \delta} \tanh \left(\frac{\sqrt{\theta^{2}-4 \delta}}{2}(q+\Sigma)\right)-\frac{\theta}{2 \delta}\right)^{2},
\end{aligned}
$$

where

$$
q=k\left(x+\frac{1}{\Gamma(\mu)}\right)^{\mu}-k \sqrt{-\gamma k^{2} \theta^{2}+4 \delta \gamma k^{2}-b}\left(t+\frac{1}{\Gamma(\mu)}\right)^{\mu} .
$$

According to Family II, (22) can be written as

$$
\begin{aligned}
\Psi_{14}(q)= & -\frac{6 k^{2} \gamma \delta}{\beta} \\
& -6 \frac{k^{2} \gamma \theta}{\beta}\left(\frac{\sqrt{-\theta^{2}+4 \delta}}{2 \delta} \tan \left(\frac{\sqrt{-\theta^{2}+4 \delta}}{2}(q+\Sigma)\right)-\frac{\theta}{2 \delta}\right) \\
& -6 \frac{k^{2} \gamma}{\beta}\left(\frac{\sqrt{\theta^{2}-4 \delta}}{2 \delta} \tan \left(\frac{\sqrt{-\theta^{2}+4 \delta}}{2}(q+\Sigma)\right)-\frac{\theta}{2 \delta}\right)^{2},
\end{aligned}
$$

where

$$
q=k\left(x+\frac{1}{\Gamma(\mu)}\right)^{\mu}-k \sqrt{-\gamma k^{2} \theta^{2}+4 \delta \gamma k^{2}-b}\left(t+\frac{1}{\Gamma(\mu)}\right)^{\mu} .
$$

According to Family III, (22) becomes

$$
\begin{aligned}
\Psi_{15}(q)= & -6 \frac{k^{2} \gamma \theta}{\beta}\left(\frac{\exp (\theta(q+\Sigma))-1}{\theta}\right) \\
& -6 \frac{k^{2} \gamma}{\beta}\left(\frac{\exp (\theta(q+\Sigma))-1}{\theta}\right)^{2},
\end{aligned}
$$


where

$$
q=k\left(x+\frac{1}{\Gamma(\alpha)}\right)^{\alpha}-k \sqrt{-\gamma k^{2} \theta^{2}-b}\left(t+\frac{1}{\Gamma(\alpha)}\right)^{\alpha} .
$$

According to Family IV, (22) can be written as

$$
\begin{aligned}
\Psi_{16}(q)= & -\frac{6 k^{2} \gamma \delta}{\beta}-6 \frac{k^{2} \gamma \theta}{\beta}\left(-\frac{2 \theta(q+\Sigma)+4}{\theta^{2}(q+E)}\right) \\
& -6 \frac{k^{2} \gamma}{\beta}\left(-\frac{2 \theta(q+E)+4}{\theta^{2}(q+\Sigma)}\right)^{2},
\end{aligned}
$$

where

$$
q=k\left(x+\frac{1}{\Gamma(\mu)}\right)^{\mu}-k \sqrt{-b}\left(t+\frac{1}{\Gamma(\mu)}\right)^{\mu} .
$$

Fifth:

$$
\begin{aligned}
& k=\frac{1}{6} \frac{\sqrt{-6 \gamma \beta \pi_{2}}}{\delta \gamma}, \\
& \omega=\frac{1}{6} \frac{\sqrt{2} \sqrt{\delta \gamma \beta \pi_{2}\left(3 b \delta+8 \beta \pi_{2}\right)}}{\delta^{2} \gamma}, \\
& \pi_{0}=\frac{2 \pi_{2}}{\delta}, \tau_{2}=\frac{\pi_{2}}{\delta^{2}} .
\end{aligned}
$$

According to Family I, (22) becomes

$$
\begin{aligned}
\Psi_{17}(q)= & \frac{2 \pi_{2}}{\delta}-\pi_{2} \frac{\delta}{\sqrt{-\delta}} \operatorname{coth}^{2}\left(\frac{\sqrt{-4 \delta}}{2}(q+\Sigma)\right) \\
& -\frac{\pi_{2}}{\delta^{2}} \frac{\sqrt{-\delta}}{\delta} \tanh ^{2}\left(\frac{\sqrt{-4 \delta}}{2}(q+\Sigma)\right),
\end{aligned}
$$

where

$$
\begin{aligned}
q= & \frac{1}{6} \frac{\sqrt{-6 \gamma \beta \pi_{2}}}{\delta \gamma}\left(x+\frac{1}{\Gamma(\mu)}\right)^{\mu} \\
& -\frac{1}{6} \frac{\sqrt{2} \sqrt{\delta \gamma \beta \pi_{2}\left(3 b \delta+8 \beta \pi_{2}\right)}}{\delta^{2} \gamma}\left(t+\frac{1}{\Gamma(\mu)}\right)^{\mu} .
\end{aligned}
$$

According to Family II, (22) becomes

$$
\begin{gathered}
\Psi_{18}(q)=\frac{2 \pi_{2}}{\delta}+\pi_{2} \frac{2 \delta}{\sqrt{4 \delta}} \cot ^{2}\left(\frac{\sqrt{4 \delta}}{2}(q+\Sigma)\right) \\
+\frac{\pi_{2}}{\delta^{2}} \frac{\sqrt{4 \delta}}{2 \delta} \tan ^{2}\left(\frac{\sqrt{4 \delta}}{2}(q+\Sigma)\right),
\end{gathered}
$$

where

$$
\begin{aligned}
q= & \frac{1}{6} \frac{\sqrt{-6 \gamma \beta \pi_{2}}}{\delta \gamma}\left(x+\frac{1}{\Gamma(\mu)}\right)^{\mu} \\
& -\frac{1}{6} \frac{\sqrt{2} \sqrt{\delta \gamma \beta \pi_{2}\left(3 b \delta+8 \beta \pi_{2}\right)}}{\delta^{2} \gamma}\left(t+\frac{1}{\Gamma(\mu)}\right)^{\mu} .
\end{aligned}
$$

\section{The Second Equation}

By utilizing the following transformation:

$$
q=k\left(x+\frac{1}{\Gamma(\mu)}\right)^{\mu}+c\left(y+\frac{1}{\Gamma(\mu)}\right)^{\mu}-\omega\left(t+\frac{1}{\Gamma(\mu)}\right)^{\mu}
$$

then, equation (1) transformed to

$$
-c \Psi^{\prime}+a k^{2} \omega \Psi^{\prime \prime \prime}+4 a k \Psi \Omega^{\prime}+4 a k \Psi^{\prime} \Omega=0,
$$

$$
\omega \Psi^{\prime}-k \Omega^{\prime}=0
$$

where $\Psi^{\prime}=d \Psi / d q$ and $\Omega^{\prime}=d \Omega / d q$. By putting $\Omega=(\omega / k) \Psi$ into equation (66), it can be seen as

$$
-c \Psi^{\prime}+a k^{2} \Psi \Psi^{\prime \prime \prime}+8 a \omega \Psi \Psi^{\prime}=0 .
$$

The balance number will be obtained $\chi=2$ by using the balance principle. Then, the exact solution is given as

$$
\Psi(q)=\pi_{0}+\pi_{1} Y(q)+\pi_{2} Y^{2}(q)+\frac{\tau_{1}}{Y(q)}+\frac{\tau_{2}}{Y^{2}(q)}
$$

Firstly, we substitute the expressions of $\Psi(q)$ in (69) into (68) and collect all terms with the same order of $Y(q)$. Then, by equating the coefficient of each polynomial to zero, we obtain a set of algebraic equations as follows:

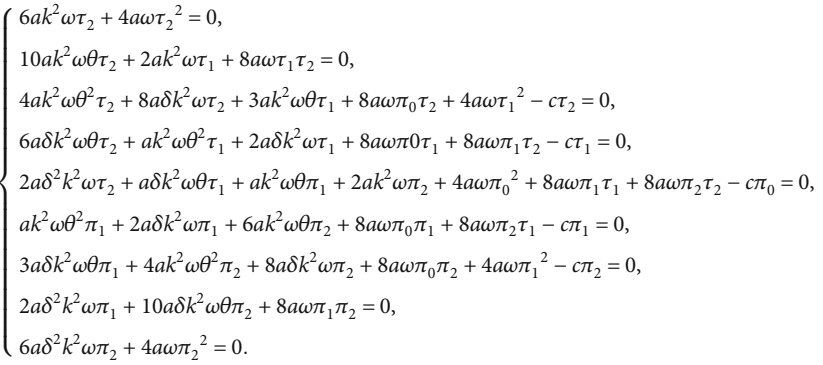

First:

$$
\begin{aligned}
c= & -\left(a k^{2} \theta^{2}-4 a \delta k^{2}\right) \omega, \pi_{0}=-\frac{1}{4} k^{2} \theta^{2} \\
& -\frac{1}{2} \delta k^{2}, \tau_{1}=-\frac{3}{2} \theta k^{2}, \tau_{2}=-\frac{3}{2} k^{2} .
\end{aligned}
$$


According to Family I, (69) can be written as

$$
\begin{aligned}
\Psi_{1}(q)= & -\frac{1}{4} k^{2} \theta^{2}-\frac{1}{2} \delta k^{2} \\
& -\frac{(3 / 2) \theta k^{2}}{\left(-\left(\sqrt{\theta^{2}-4 \delta} / 2 \delta\right) \tanh \left(\left(\sqrt{\theta^{2}-4 \delta} / 2\right)(q+\Sigma)\right)-(\theta / 2 \delta)\right)} \\
& -\frac{(3 / 2) k^{2}}{\left(-\left(\sqrt{\theta^{2}-4 \delta} / 2 \delta\right) \tanh \left(\left(\sqrt{\theta^{2}-4 \delta} / 2\right)(q+\Sigma)\right)-(\theta / 2 \delta)\right)^{2}},
\end{aligned}
$$

where

$$
\begin{aligned}
q= & k\left(x+\frac{1}{\Gamma(\mu)}\right)^{\mu}-\left(a k^{2} \theta^{2}-4 a \delta k^{2}\right) \omega\left(y+\frac{1}{\Gamma(\mu)}\right)^{\mu} \\
& -\omega\left(t+\frac{1}{\Gamma(\mu)}\right)^{\mu} .
\end{aligned}
$$

According to Family II, (69) becomes

$$
\begin{aligned}
\Psi_{2}(q)= & -\frac{1}{4} k^{2} \theta^{2}-\frac{1}{2} \delta k^{2} \\
& -\frac{(3 / 2) \theta k^{2}}{\left(\left(\sqrt{\theta^{2}-4 \delta} / 2 \delta\right) \tan \left(\left(\sqrt{-\theta^{2}+4 \delta} / 2\right)(q+\Sigma)\right)-(\theta / 2 \delta)\right)} \\
& -\frac{(3 / 2) k^{2}}{\left(\left(\sqrt{\theta^{2}-4 \delta} / 2 \delta\right) \tan \left(\left(\sqrt{-\theta^{2}+4 \delta} / 2\right)(q+\Sigma)\right)-(\theta / 2 \delta)\right)^{2}},
\end{aligned}
$$

where

$$
\begin{aligned}
q= & k\left(x+\frac{1}{\Gamma(\mu)}\right)^{\mu}-\left(a k^{2} \theta^{2}-4 a \delta k^{2}\right) \omega\left(y+\frac{1}{\Gamma(\mu)}\right)^{\mu} \\
& -\omega\left(t+\frac{1}{\Gamma(\mu)}\right)^{\mu} .
\end{aligned}
$$

According to Family III, (69) becomes

$$
\begin{aligned}
\Psi_{3}(q)= & -\frac{1}{4} k^{2} \theta^{2}-\frac{(3 / 2) \theta k^{2}}{((\exp (\theta(q+\Sigma))-1) / \theta)} \\
& -\frac{(3 / 2) k^{2}}{((\exp (\theta(q+\Sigma))-1) / \theta)^{2}},
\end{aligned}
$$

where

$$
q=k\left(x+\frac{1}{\Gamma(\mu)}\right)^{\mu}-a k^{2} \theta^{2} \omega\left(y+\frac{1}{\Gamma(\mu)}\right)^{\mu}-\omega\left(t+\frac{1}{\Gamma(\mu)}\right)^{\mu}
$$

Second:

$$
c=-\left(-a k^{2} \theta^{2}+4 a \delta k^{2}\right) \omega, \pi_{0}=-\frac{3}{2} \delta k^{2}, \tau_{1}=-\frac{3}{2} \theta k^{2}, \tau_{2}=-\frac{3}{2} k^{2} .
$$

According to Family I, (69) can be seen as

$$
\begin{aligned}
\Psi_{4}(q)= & -\frac{3}{2} \delta k^{2} \\
& -\frac{(3 / 2) \theta k^{2}}{\left(-\left(\sqrt{\theta^{2}-4 \delta} / 2 \delta\right) \tanh \left(\left(\sqrt{\theta^{2}-4 \delta} / 2\right)(q+\Sigma)\right)-(\theta / 2 \delta)\right)} \\
& -\frac{(3 / 2) k^{2}}{\left(-\left(\sqrt{\theta^{2}-4 \delta} / 2 \delta\right) \tanh \left(\left(\sqrt{\theta^{2}-4 \delta} / 2\right)(q+\Sigma)\right)-(\theta / 2 \delta)\right)^{2}},
\end{aligned}
$$

where

$$
\begin{aligned}
q= & k\left(x+\frac{1}{\Gamma(\mu)}\right)^{\mu}-\left(-a k^{2} \theta^{2}+4 a \delta k^{2}\right) \omega\left(y+\frac{1}{\Gamma(\mu)}\right)^{\mu} \\
& -\omega\left(t+\frac{1}{\Gamma(\mu)}\right)^{\mu} .
\end{aligned}
$$

According to Family II, (69) becomes

$$
\begin{aligned}
& \Psi_{5}(q)=-\frac{3}{2} \delta k^{2} \\
& -\frac{(3 / 2) \theta k^{2}}{\left(\left(\sqrt{\theta^{2}-4 \delta} / 2 \delta\right) \tan \left(\left(\sqrt{-\theta^{2}+4 \delta} / 2\right)(q+\Sigma)\right)-(\theta / 2 \delta)\right)} \\
& -\frac{(3 / 2) k^{2}}{\left(\left(\sqrt{\theta^{2}-4 \delta} / 2 \delta\right) \tan \left(\left(\sqrt{-\theta^{2}+4 \delta} / 2\right)(q+\Sigma)\right)-(\theta / 2 \delta)\right)^{2}},
\end{aligned}
$$

where

$$
\begin{aligned}
q= & k\left(x+\frac{1}{\Gamma(\mu)}\right)^{\mu}-\left(-a k^{2} \theta^{2}+4 a \delta k^{2}\right) \omega\left(y+\frac{1}{\Gamma(\mu)}\right)^{\mu} \\
& -\omega\left(t+\frac{1}{\Gamma(\mu)}\right)^{\mu} .
\end{aligned}
$$

According to Family III, (69) can be written as

$$
\begin{aligned}
\Psi_{6}(q)= & -\frac{(3 / 2) \theta k^{2}}{((\exp (\theta(q+\Sigma))-1) / \theta)} \\
& -\frac{(3 / 2) k^{2}}{((\exp (\theta(q+\Sigma))-1) / \theta)^{2}},
\end{aligned}
$$

where

$$
q=k\left(x+\frac{1}{\Gamma(\mu)}\right)^{\mu}+a k^{2} \theta^{2} \omega\left(y+\frac{1}{\Gamma(\mu)}\right)^{\mu}-\omega\left(t+\frac{1}{\Gamma(\mu)}\right)^{\mu} .
$$


Third:

$$
\begin{aligned}
c & =-\left(a k^{2} \theta^{2}-4 a \delta k^{2}\right) \omega, \pi_{0}=-\frac{1}{4} k^{2} \theta^{2}-\frac{1}{2} \delta k^{2}, \pi_{1} \\
& =-\frac{3}{2} \theta k^{2}, \pi_{2}=-\frac{3}{2} k^{2} .
\end{aligned}
$$

According to Family I, (69) becomes

$$
\begin{aligned}
\Psi_{7}(q)= & -\frac{1}{4} k^{2} \theta^{2}-\frac{1}{2} \delta k^{2}-\frac{3}{2} \theta k^{2} \\
& \cdot\left(-\frac{\sqrt{\theta^{2}-4 \delta}}{2 \delta} \tanh \left(\frac{\sqrt{\theta^{2}-4 \delta}}{2}(q+\Sigma)\right)-\frac{\theta}{2 \delta}\right) \\
& -\frac{3}{2} k^{2}\left(-\frac{\sqrt{\theta^{2}-4 \delta}}{2 \delta} \tanh \left(\frac{\sqrt{\theta^{2}-4 \delta}}{2}(q+\Sigma)\right)-\frac{\theta}{2 \delta}\right)^{2},
\end{aligned}
$$

where

$$
\begin{aligned}
q= & k\left(x+\frac{1}{\Gamma(\mu)}\right)^{\mu}-\left(a k^{2} \theta^{2}-4 a \delta k^{2}\right) \omega\left(y+\frac{1}{\Gamma(\mu)}\right)^{\mu} \\
& -\omega\left(t+\frac{1}{\Gamma(\mu)}\right)^{\mu} .
\end{aligned}
$$

According to Family II, (69) can be written as

$$
\begin{aligned}
\Psi_{8}(q)= & -\frac{1}{4} k^{2} \theta^{2}-\frac{1}{2} \delta k^{2} \\
& -\frac{3}{2} \theta k^{2}\left(\frac{\sqrt{\theta^{2}-4 \delta}}{2 \delta} \tan \left(\frac{\sqrt{-\theta^{2}+4 \delta}}{2}(q+\Sigma)\right)-\frac{\theta}{2 \delta}\right) \\
& -\frac{3}{2} k^{2}\left(\frac{\sqrt{\theta^{2}-4 \delta}}{2 \delta} \tan \left(\frac{\sqrt{-\theta^{2}+4 \delta}}{2}(q+\Sigma)\right)-\frac{\theta}{2 \delta}\right)^{2},
\end{aligned}
$$

where

$$
\begin{aligned}
q= & k\left(x+\frac{1}{\Gamma(\mu)}\right)^{\mu}-\left(a k^{2} \theta^{2}-4 a \delta k^{2}\right) \omega\left(y+\frac{1}{\Gamma(\mu)}\right)^{\mu} \\
& -\omega\left(t+\frac{1}{\Gamma(\mu)}\right)^{\mu} .
\end{aligned}
$$

According to Family III, (69) can be written as

$$
\begin{aligned}
\Psi_{9}(q)= & -\frac{1}{4} k^{2} \theta^{2}-\frac{(3 / 2) \theta k^{2}}{(\theta /(\exp (\theta(q+\Sigma))-1))} \\
& -\frac{(3 / 2) k^{2}}{(\theta /(\exp (\theta(q+\Sigma))-1))^{2}},
\end{aligned}
$$

where

$$
q=k\left(x+\frac{1}{\Gamma(\mu)}\right)^{\mu}-a k^{2} \theta^{2} \omega\left(y+\frac{1}{\Gamma(\mu)}\right)^{\mu}-\omega\left(t+\frac{1}{\Gamma(\mu)}\right)^{\mu} .
$$

Fourth:

$$
\begin{aligned}
& c=-\left(-a k^{2} \theta^{2}+4 a \delta k^{2}\right) \omega, \\
& \pi_{0}=-\frac{3}{2} \delta k^{2}, \pi_{1}=-\frac{3}{2} \theta k^{2}, \pi_{2}=-\frac{3}{2} k^{2} .
\end{aligned}
$$

According to Family I, (69) can be seen as

$$
\begin{aligned}
\Psi_{10}(q)= & -\frac{3}{2} \delta k^{2}-\frac{3}{2} \theta k^{2} \\
& \cdot\left(-\frac{\sqrt{\theta^{2}-4 \delta}}{2 \delta} \tanh \left(\frac{\sqrt{\theta^{2}-4 \delta}}{2}(q+\Sigma)\right)-\frac{\theta}{2 \delta}\right) \\
& -\frac{3}{2} k^{2}\left(-\frac{\sqrt{\theta^{2}-4 \delta}}{2 \delta} \tanh \left(\frac{\sqrt{\theta^{2}-4 \delta}}{2}(q+\Sigma)\right)-\frac{\theta}{2 \delta}\right)^{2},
\end{aligned}
$$

where

$$
\begin{aligned}
q= & k\left(x+\frac{1}{\Gamma(\mu)}\right)^{\mu}-\left(-a k^{2} \theta^{2}+4 a \delta k^{2}\right) \omega\left(y+\frac{1}{\Gamma(\mu)}\right)^{\mu} \\
& -\omega\left(t+\frac{1}{\Gamma(\mu)}\right)^{\mu} .
\end{aligned}
$$

According to Family II, (69) can be written as

$$
\begin{aligned}
\Psi_{11}(q)= & -\frac{3}{2} \delta k^{2} \\
& -\frac{3}{2} \theta k^{2}\left(\frac{\sqrt{\theta^{2}-4 \delta}}{2 \delta} \tan \left(\frac{\sqrt{-\theta^{2}+4 \delta}}{2}(q+\Sigma)\right)-\frac{\theta}{2 \delta}\right) \\
& -\frac{3}{2} k^{2}\left(\frac{\sqrt{\theta^{2}-4 \delta}}{2 \delta} \tan \left(\frac{\sqrt{-\theta^{2}+4 \delta}}{2}(q+\Sigma)\right)-\frac{\theta}{2 \delta}\right)^{2},
\end{aligned}
$$

where

$$
\begin{aligned}
q= & k\left(x+\frac{1}{\Gamma(\alpha)}\right)^{\alpha}-\left(-a k^{2} \theta^{2}+4 a \delta k^{2}\right) \omega\left(y+\frac{1}{\Gamma(\mu)}\right)^{\mu} \\
& -\omega\left(t+\frac{1}{\Gamma(\mu)}\right)^{\mu} .
\end{aligned}
$$

According to Family III, (69) becomes

$$
\begin{aligned}
\Psi_{12}(q)= & -\frac{(3 / 2) \theta k^{2}}{(\theta /(\exp (\theta(q+\Sigma))-1))} \\
& -\frac{3 / 2 k^{2}}{(\theta /(\exp (\theta(q+\Sigma))-1))^{2}},
\end{aligned}
$$


where

$$
q=k\left(x+\frac{1}{\Gamma(\mu)}\right)^{\mu}+a k^{2} \theta^{2} \omega\left(y+\frac{1}{\Gamma(\mu)}\right)^{\mu}-\omega\left(t+\frac{1}{\Gamma(\mu)}\right)^{\mu} .
$$

Fifth:

$$
k=\frac{1}{4} \frac{\sqrt{-a \delta \omega c}}{a \delta \omega}, \pi_{0}=\frac{3}{16} \frac{c}{a \omega}, \pi_{2}=\frac{3 c \delta}{32 a \omega}, \tau_{2}=\frac{3 c}{32 a \delta \omega} .
$$

According to Family I, (22) can be written as

$$
\begin{aligned}
\Psi_{13}(q)= & \frac{3}{16} \frac{c}{a \omega}-\frac{3 c \delta}{32 a \omega} \frac{\delta}{\sqrt{-\delta}} \operatorname{coth}^{2}\left(\frac{\sqrt{-4 \delta}}{2}(q+\Sigma)\right) \\
& -\frac{3 c}{32 a \delta \omega} \frac{\sqrt{-\delta}}{\delta} \tanh ^{2}\left(\frac{\sqrt{-4 \delta}}{2}(q+\Sigma)\right),
\end{aligned}
$$

where

$$
\begin{aligned}
q= & \frac{1}{4} \frac{\sqrt{-a \delta \omega c}}{a \delta \omega}\left(x+\frac{1}{\Gamma(\alpha)}\right)^{\alpha}-c\left(y+\frac{1}{\Gamma(\alpha)}\right)^{\alpha} \\
& -\omega\left(t+\frac{1}{\Gamma(\alpha)}\right)^{\alpha} .
\end{aligned}
$$

According to Family II, (22) becomes

$$
\begin{aligned}
\Psi_{14}(q)= & \frac{3}{16} \frac{c}{a \omega}+\frac{3 c \delta}{32 a \omega} \frac{\delta}{\sqrt{\delta}} \cot ^{2}\left(\frac{\sqrt{4 \delta}}{2}(q+\Sigma)\right) \\
& +\frac{3 c}{32 a \delta \omega} \frac{\sqrt{\delta}}{\delta} \tan ^{2}\left(\frac{\sqrt{4 \delta}}{2}(q+\Sigma)\right),
\end{aligned}
$$

where

$$
\begin{aligned}
q= & \frac{1}{4} \frac{\sqrt{-a \delta \omega c}}{a \delta \omega}\left(x+\frac{1}{\Gamma(\mu)}\right)^{\mu}-c\left(y+\frac{1}{\Gamma(\mu)}\right)^{\mu} \\
& -\omega\left(t+\frac{1}{\Gamma(\mu)}\right)^{\mu} .
\end{aligned}
$$

Sixth:

$$
k=\frac{1}{4} \frac{\sqrt{a \delta \omega c}}{a \delta \omega}, \pi_{0}=\frac{1}{16} \frac{c}{a \omega}, \pi_{2}=-\frac{3 c \delta}{32 a \omega}, \tau_{2}=-\frac{3 c}{32 a \delta \omega} .
$$

According to Family I, (22) can be written as

$$
\begin{aligned}
\Psi_{15}(q)= & \frac{1}{16} \frac{c}{a \omega}+\frac{3 c \delta}{32 a \omega} \frac{\delta}{\sqrt{-\delta}} \operatorname{coth}^{2}\left(\frac{\sqrt{-4 \delta}}{2}(q+\Sigma)\right) \\
& +\frac{3 c}{32 a \delta \omega} \frac{\sqrt{-\delta}}{\delta} \tanh ^{2}\left(\frac{\sqrt{-4 \delta}}{2}(q+\Sigma)\right)
\end{aligned}
$$

where

$$
\begin{aligned}
q= & \frac{1}{4} \frac{\sqrt{a \delta \omega c}}{a \delta \omega}\left(x+\frac{1}{\Gamma(\alpha)}\right)^{\alpha}-c\left(y+\frac{1}{\Gamma(\alpha)}\right)^{\alpha} \\
& -\omega\left(t+\frac{1}{\Gamma(\alpha)}\right)^{\alpha} .
\end{aligned}
$$

According to Family II, (22) can be seen as

$$
\begin{aligned}
\Psi_{16}(q)= & \frac{1}{16} \frac{c}{a \omega}-\frac{3 c \delta}{32 a \omega} \frac{\delta}{\sqrt{\delta}} \cot ^{2}\left(\frac{\sqrt{4 \delta}}{2}(q+\Sigma)\right) \\
& -\frac{3 c}{32 a \delta \omega} \frac{\sqrt{\delta}}{\delta} \tan ^{2}\left(\frac{\sqrt{4 \delta}}{2}(q+\Sigma)\right),
\end{aligned}
$$

where

$$
\begin{aligned}
q= & \frac{1}{4} \frac{\sqrt{a \delta \omega c}}{a \delta \omega}\left(x+\frac{1}{\Gamma(\mu)}\right)^{\mu}-c\left(y+\frac{1}{\Gamma(\mu)}\right)^{\mu} \\
& -\omega\left(t+\frac{1}{\Gamma(\mu)}\right)^{\mu} .
\end{aligned}
$$

\section{The Third Equation}

By utilizing the following transformation:

$$
\eta=k\left(x+\frac{1}{\Gamma(\mu)}\right)^{\mu}-\omega\left(t+\frac{1}{\Gamma(\mu)}\right)^{\mu},
$$

then, equation (1) transformed to

$$
2\left(\omega^{2}+k^{2}\right) \Psi+k \omega \Psi^{2}+2 k^{2} \omega^{2} \Psi^{\prime \prime}=0,
$$

where $\Psi^{\prime}=d \Psi / d q$, and by integrating equation (110) twice with respect to $q$, it can be seen as

$$
\left(\omega^{2}+b k^{2}\right) \Psi+\beta k^{2} \Psi^{2}+\gamma k^{4} \Psi^{\prime \prime}=0 .
$$

The balance number will be obtained $\chi=2$ by using the balance principle. Then, the exact solution is given as

$$
\Psi(q)=\pi_{0}+\pi_{1} Y(q)+\pi_{2} Y^{2}(q)+\frac{\tau_{1}}{Y(q)}+\frac{\tau_{2}}{Y^{2}(q)} .
$$

Firstly, we substitute the expressions of $\Psi(q)$ in (112) into (111) and collect all terms with the same order of $Y(q)$. Then, 
by equating the coefficient of each polynomial to zero, we obtain a set of algebraic equations as follows:

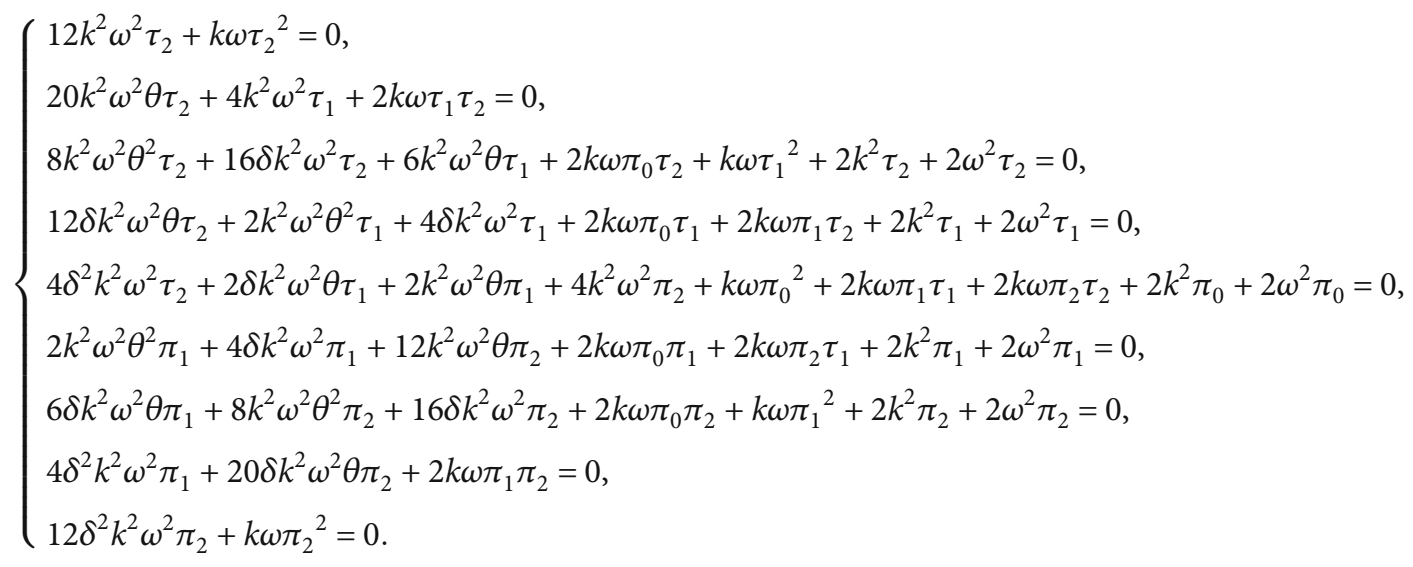

First:

$$
\begin{aligned}
\delta & =\frac{1}{16} \frac{k^{2}+\omega^{2}}{k^{2} \omega^{2}}, \theta=0, \pi_{0}=-\frac{3}{2} \frac{k^{2}+\omega^{2}}{k \omega}, \pi_{2} \\
& =-\frac{3\left(k^{2}+\omega^{2}\right)^{2}}{64 k^{3} \omega^{3}}, \tau_{2}=-12 k \omega .
\end{aligned}
$$

According to Family I, (112) can be written as

$$
\begin{aligned}
\Psi_{1}(q)= & -\frac{3 k^{2}+\omega^{2}}{k \omega}+\frac{3\left(k^{2}+\omega^{2}\right)^{2}}{64 k^{3} \omega^{3}} \delta \operatorname{coth}^{2}\left(\frac{\sqrt{-4 \delta}}{2}(q+\Sigma)\right) \\
& +\frac{12 k \omega}{\delta} \tanh ^{2}\left(\frac{\sqrt{-4 \delta}}{2}(q+\Sigma)\right),
\end{aligned}
$$

where

$$
q=k\left(x+\frac{1}{\Gamma(\alpha)}\right)^{\alpha}-\omega\left(t+\frac{1}{\Gamma(\alpha)}\right)^{\alpha}
$$

According to Family II, (112) becomes

$$
\begin{aligned}
\Psi_{2}(q)= & -\frac{3}{2} \frac{k^{2}+\omega^{2}}{k \omega}-\frac{3\left(k^{2}+\omega^{2}\right)^{2}}{64 k^{3} \omega^{3}} \delta \cot ^{2}\left(\frac{\sqrt{4 \delta}}{2}(q+\Sigma)\right) \\
& +-\frac{12 k \omega}{\delta} \tan ^{2}\left(\frac{\sqrt{4 \delta}}{2}(q+\Sigma)\right)
\end{aligned}
$$

where

$$
\delta=\frac{1}{16} \frac{k^{2}+\omega^{2}}{k^{2} \omega^{2}}, q=k\left(x+\frac{1}{\Gamma(\mu)}\right)^{\mu}-\omega\left(t+\frac{1}{\Gamma(\mu)}\right)^{\mu} .
$$

Second:

$$
\begin{gathered}
\delta=-\frac{1}{16} \frac{k^{2}+\omega^{2}}{k^{2} \omega^{2}}, \theta=0, \pi_{0}=-\frac{1}{2} \frac{k^{2}+\omega^{2}}{k \omega}, \\
\pi_{2}=-\frac{3\left(k^{2}+\omega^{2}\right)^{2}}{64 k^{3} \omega^{3}}, \tau_{2}=-12 k \omega .
\end{gathered}
$$

According to Family I, (112) becomes

$$
\begin{aligned}
\Psi_{3}(q)= & -\frac{1}{2} \frac{k^{2}+\omega^{2}}{k \omega}+\frac{3\left(k^{2}+\omega^{2}\right)^{2}}{64 k^{3} \omega^{3}} \delta \operatorname{coth}^{2}\left(\frac{\sqrt{-4 \delta}}{2}(q+\Sigma)\right) \\
& +\frac{12 k \omega}{\delta} \tanh ^{2}\left(\frac{\sqrt{-4 \delta}}{2}(q+\Sigma)\right),
\end{aligned}
$$

where

$$
q=k\left(x+\frac{1}{\Gamma(\alpha)}\right)^{\alpha}-\omega\left(t+\frac{1}{\Gamma(\alpha)}\right)^{\alpha} .
$$

According to Family II, (112) can be written as

$$
\begin{aligned}
\Psi_{4}(q)= & -\frac{1}{2} \frac{k^{2}+\omega^{2}}{k \omega}-\frac{3\left(k^{2}+\omega^{2}\right)^{2}}{64 k^{3} \omega^{3}} \delta \cot ^{2}\left(\frac{\sqrt{4 \delta}}{2}(q+\Sigma)\right) \\
& +-\frac{12 k \omega}{\delta} \tan ^{2}\left(\frac{\sqrt{4 \delta}}{2}(q+\Sigma)\right),
\end{aligned}
$$




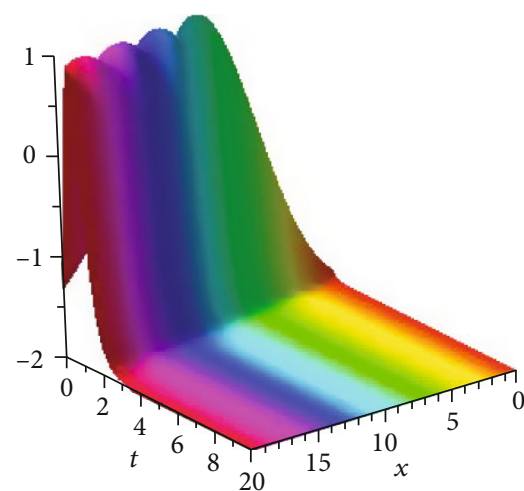

(a)

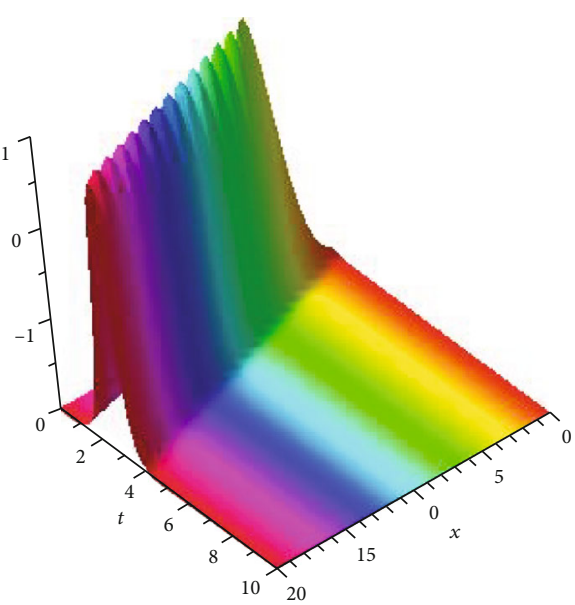

(b)

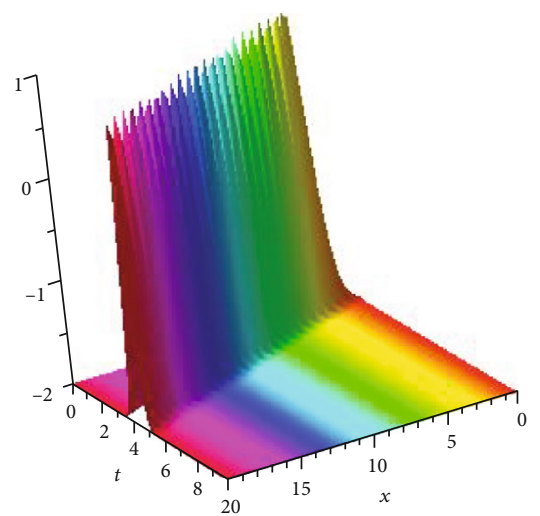

(c)

Figure 1: The different types of graphs to bright solution (25) for the parameters $\theta=3, \delta=2, k=-2, \gamma=1, b=-10$, and $\beta=2$ with providing amounts of (a) $\mu=0.5$, (b) $\mu=0.75$, and (c) $\mu=1$ within the interval $0 \leq x \leq 20,0 \leq t \leq 10$.

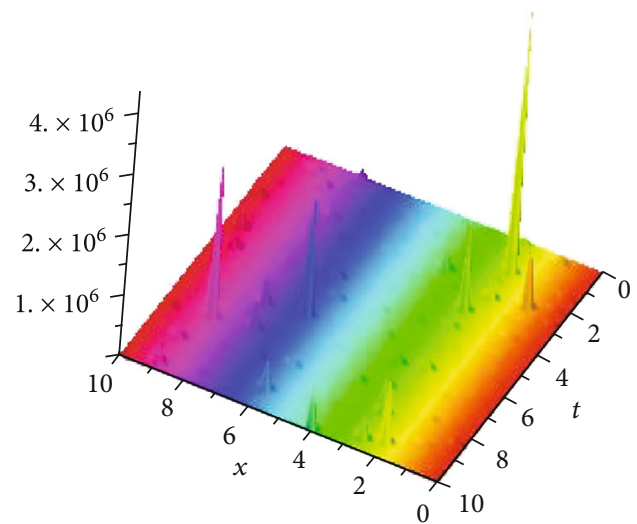

(a)

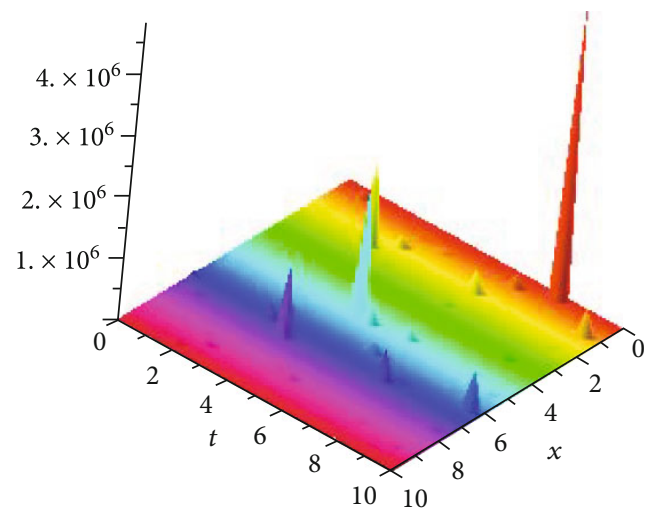

(b)

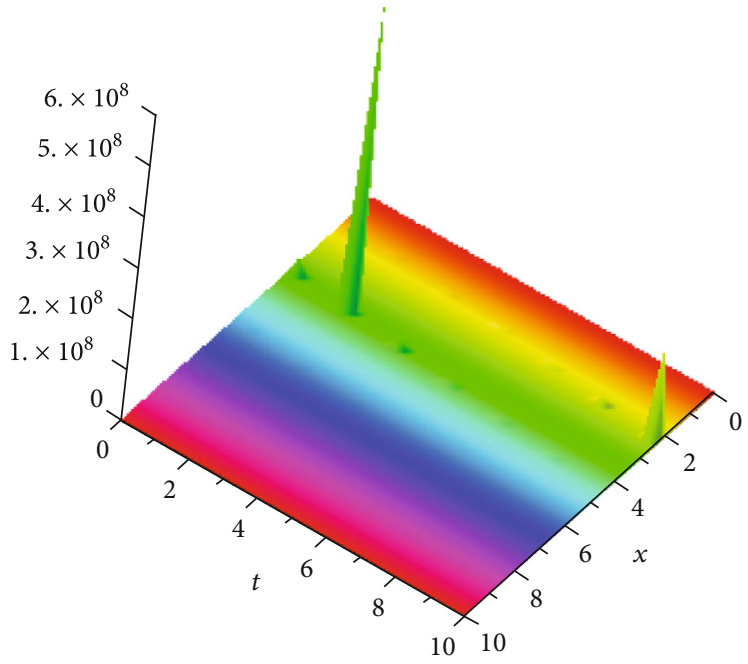

(c)

FIgURE 2: The different types of graphs to periodic solution (27) for the parameters $\theta=2, \delta=3, k=-2, \gamma=-1, b=1$, and $\beta=2$ with providing amounts of (a) $\mu=0.5$, (b) $\mu=0.75$, and (c) $\mu=1$ within the interval $0 \leq x \leq 20,0 \leq t \leq 10$. 


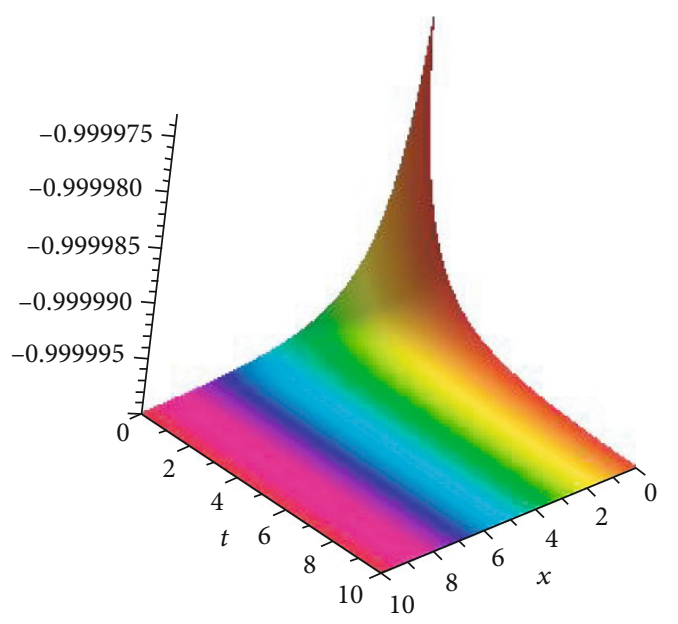

(a)

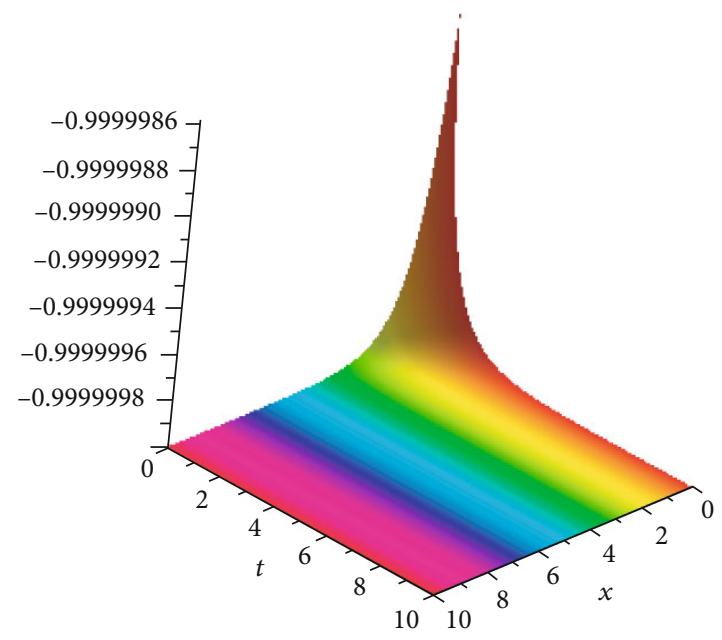

(b)

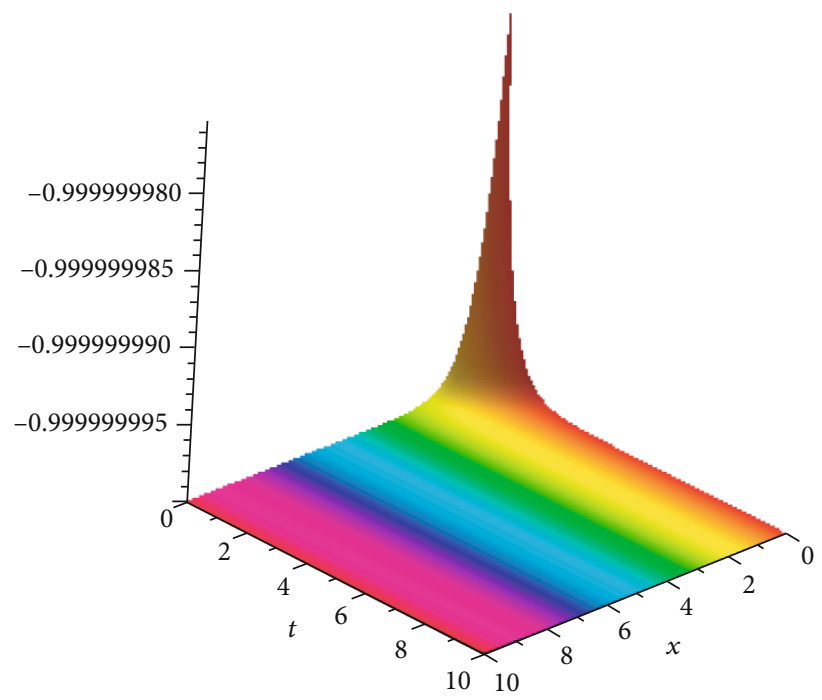

(c)

Figure 3: The different types of graphs to kink solution (72) for the parameters $\theta=3, \delta=2, d=1, k=-2, a=1, b=-10, \omega=2$, and $y=1$ with providing amounts of (a) $\mu=0.5$, (b) $\mu=0.75$, and (c) $\mu=1$ within the interval $0 \leq x, t \leq 10$.

where

$$
\delta=-\frac{1}{16} \frac{k^{2}+\omega^{2}}{k^{2} \omega^{2}}, q=k\left(x+\frac{1}{\Gamma(\mu)}\right)^{\mu}-\omega\left(t+\frac{1}{\Gamma(\mu)}\right)^{\mu} .
$$

Third:

$$
\begin{aligned}
& \delta=\frac{1}{4} \frac{k^{2} \omega^{2} \theta^{2}+k^{2}+\omega^{2}}{k^{2} \omega^{2}}, \\
& \theta=0, \pi_{0}=-3 \frac{k^{2} \omega^{2} \theta^{2}+k^{2}+\omega^{2}}{k \omega}, \pi_{1}=-3 \frac{\left(k^{2} \omega^{2} \theta^{2}+k^{2}+\omega^{2}\right) \theta}{k \omega}, \\
& \pi_{2}=-\frac{3}{4} \frac{\left(k^{2} \omega^{2} \theta^{2}+k^{2}+\omega^{2}\right)^{2}}{k^{3} \omega^{3}} .
\end{aligned}
$$

According to Family II, (112) can be seen as

$$
\begin{aligned}
\Psi_{5}(q)= & -3 \frac{k^{2} \omega^{2} \theta^{2}+k^{2}+\omega^{2}}{k \omega} \\
& -\frac{3\left(\left(k^{2} \omega^{2} \theta^{2}+k^{2}+\omega^{2}\right) \theta / k \omega\right)}{\left(\left(\sqrt{-\theta^{2}+4 \delta} / 2 \delta\right) \tan \left(\left(\sqrt{-\theta^{2}+4 \delta} / 2\right)(q+\Sigma)\right)-(\theta / 2 \delta)\right)} \\
& -\frac{(3 / 4)\left(\left(k^{2} \omega^{2} \theta^{2}+k^{2}+\omega^{2}\right)^{2} / k^{3} \omega^{3}\right)}{\left(\left(\sqrt{-\theta^{2}+4 \delta} / 2 \delta\right) \tan \left(\left(\sqrt{-\theta^{2}+4 \delta} / 2\right)(q+\Sigma)\right)-(\theta / 2 \delta)\right)^{2}},
\end{aligned}
$$

where

$$
\theta^{2}-4 \delta=-\frac{k^{2}+\omega^{2}}{k^{2} \omega^{2}}<0, q=k\left(x+\frac{1}{\Gamma(\mu)}\right)^{\mu}-\omega\left(t+\frac{1}{\Gamma(\mu)}\right)^{\mu} .
$$




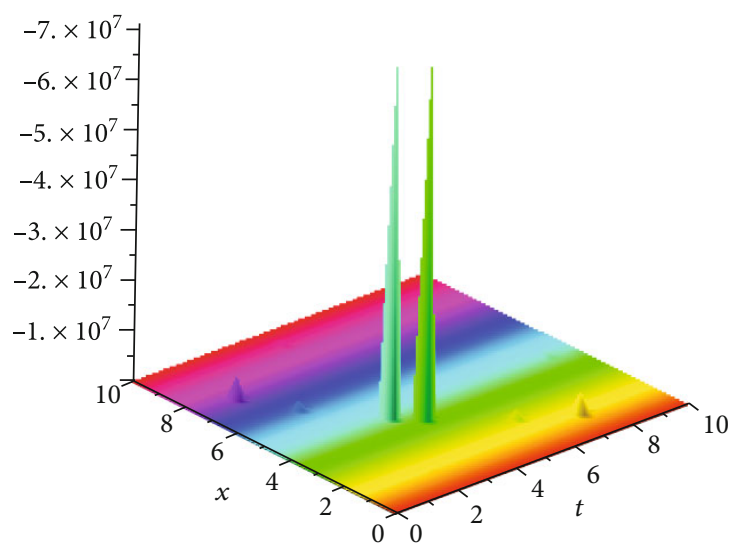

(a)

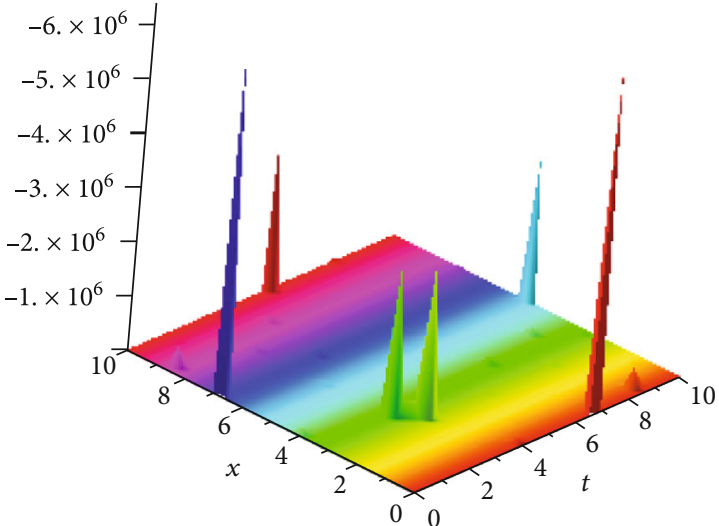

(b)

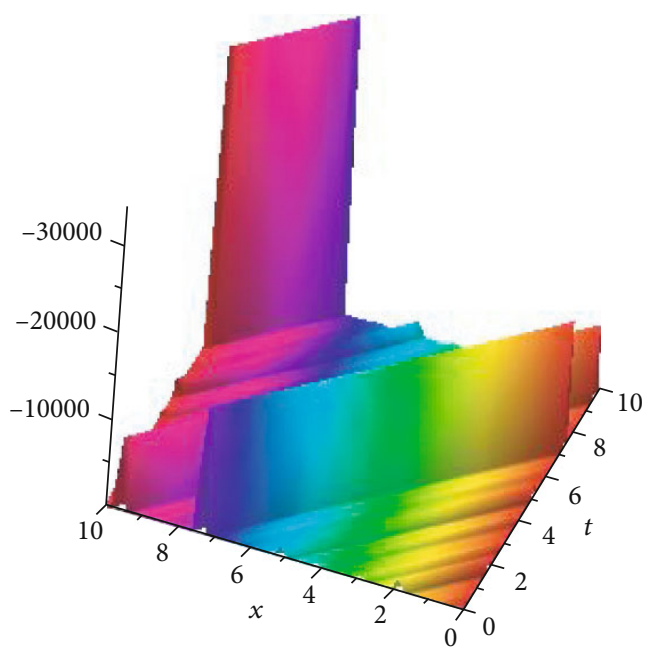

(c)

FIGURE 4: The different types of graphs to periodic solution (74) for the parameters $\theta=2, \delta=3, d=1, k=-2, a=1, b=-10, \omega=2$, and $y=1$ with providing amounts of (a) $\mu=0.5$, (b) $\mu=0.75$, and (c) $\mu=1$ within the interval $0 \leq x, t \leq 10$.

Fourth:

$$
\begin{gathered}
\delta=\frac{1}{4} \frac{k^{2} \omega^{2} \theta^{2}-k^{2}-\omega^{2}}{k^{2} \omega^{2}}, \theta=0, \pi_{0}=-\frac{3 k^{2} \omega^{2} \theta^{2}-k^{2}-\omega^{2}}{k \omega} \\
\pi_{1}=-3 \frac{\left(k^{2} \omega^{2} \theta^{2}-k^{2}-\omega^{2}\right) \theta}{k \omega} \\
\pi_{2}=-\frac{3}{4} \frac{\left(k^{2} \omega^{2} \theta^{2}-k^{2}-\omega^{2}\right)^{2}}{k^{3} \omega^{3}}
\end{gathered}
$$

According to Family I, (112) becomes

$$
\begin{aligned}
\Psi_{6}(q)= & -\frac{3 k^{2} \omega^{2} \theta^{2}-k^{2}-\omega^{2}}{k \omega} \\
& -\frac{3\left(\left(k^{2} \omega^{2} \theta^{2}-k^{2}-\omega^{2}\right) \theta / k \omega\right)}{\left(-\left(\sqrt{\theta^{2}-4 \delta} / 2 \delta\right) \tanh \left(\left(\sqrt{\theta^{2}-4 \delta} / 2\right)(q+\Sigma)\right)-(\theta / 2 \delta)\right)} \\
& -\frac{(3 / 4)\left(\left(k^{2} \omega^{2} \theta^{2}-k^{2}-\omega^{2}\right)^{2} / k^{3} \omega^{3}\right)}{\left(-\left(\sqrt{\theta^{2}-4 \delta} / 2 \delta\right) \tanh \left(\left(\sqrt{\theta^{2}-4 \delta} / 2\right)(q+\Sigma)\right)-(\theta / 2 \delta)\right)^{2}},
\end{aligned}
$$

where

$$
\begin{gathered}
\theta^{2}-4 \delta=\frac{k^{2}+\omega^{2}}{k^{2} \omega^{2}}>0, q \\
=k\left(x+\frac{1}{\Gamma(\mu)}\right)^{\mu} \\
-\omega\left(t+\frac{1}{\Gamma(\mu)}\right)^{\mu} .
\end{gathered}
$$

Fifth:

$$
\begin{aligned}
\delta= & -\frac{144 k^{2}+144 \omega^{2}-\tau_{1}{ }^{2}}{576 k^{2} \omega^{2}}, \\
\theta & =-\frac{1}{12} \frac{\tau_{1}}{k \omega}, \\
\pi_{0} & =\frac{1}{48} \frac{48 k^{2}+48 \omega^{2}-\tau_{1}{ }^{2}}{k \omega}, \\
\tau_{2} & =-12 k \omega .
\end{aligned}
$$




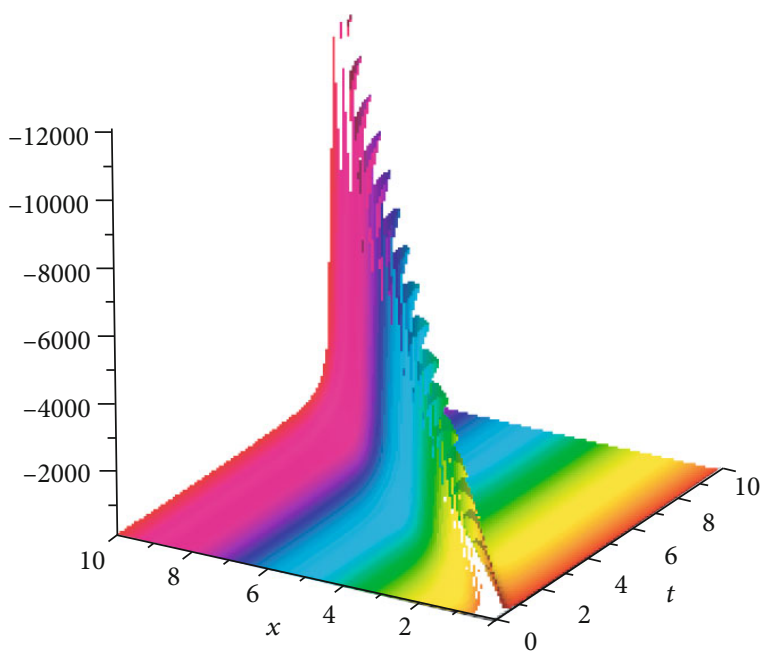

(a)

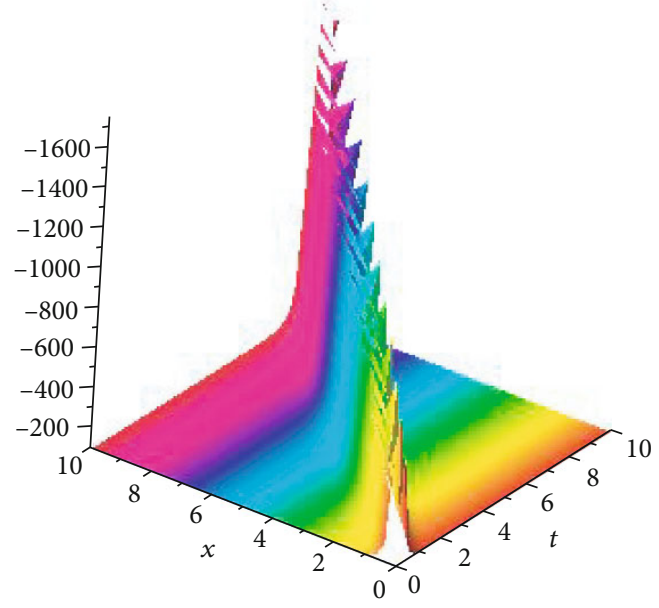

(b)

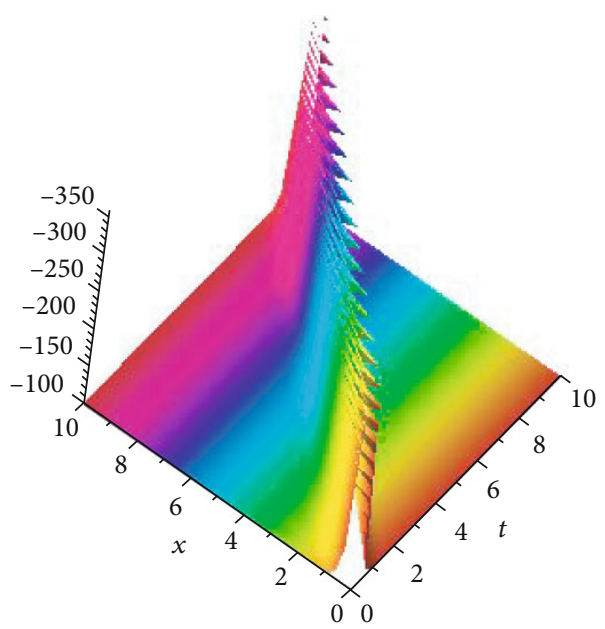

(c)

Figure 5: The different types of graphs to bright solution (115) for the parameters $\delta=-2, d=1, k=2$, and $\omega=2$ with providing amounts of (a) $\mu=0.5$, (b) $\mu=0.75$, and (c) $\mu=1$ within the interval $0 \leq x, t \leq 10$.

According to Family I, (112) can be written as

$$
\begin{aligned}
\Psi_{7}(q)= & \frac{1}{48} \frac{48 k^{2}+48 \omega^{2}-\tau_{1}^{2}}{k \omega} \\
& +\tau_{1}\left(-\frac{\sqrt{\theta^{2}-4 \delta}}{2 \delta} \tanh \left(\frac{\sqrt{\theta^{2}-4 \delta}}{2}(q+\Sigma)\right)-\frac{\theta}{2 \delta}\right) \\
& -12 k \omega\left(-\frac{\sqrt{\theta^{2}-4 \delta}}{2 \delta} \tanh \left(\frac{\sqrt{\theta^{2}-4 \delta}}{2}(q+\Sigma)\right)-\frac{\theta}{2 \delta}\right)^{2},
\end{aligned}
$$

where

$$
\begin{aligned}
\frac{\theta}{2 \delta}= & \frac{24 \tau_{1} k \omega}{144 k^{2}+144 \omega^{2}-\tau_{1}{ }^{2}}, \theta^{2}-4 \delta=\frac{k^{2}+\omega^{2}}{k^{2} \omega^{2}} \\
& >0, q=k\left(x+\frac{1}{\Gamma(\alpha)}\right)^{\alpha}-\omega\left(t+\frac{1}{\Gamma(\alpha)}\right)^{\alpha}
\end{aligned}
$$

According to Family III, (112) becomes

$$
\begin{aligned}
\Psi_{8}(q)= & \frac{1}{48} \frac{48 k^{2}+48 \omega^{2}-\tau_{1}^{2}}{k \omega}+\tau_{1}\left(\frac{\theta}{\exp (\theta(q+\Sigma))-1}\right) \\
& -12 k \omega\left(\frac{\theta}{\exp (\theta(q+\Sigma))-1}\right)^{2}
\end{aligned}
$$

where

$$
\begin{aligned}
\tau_{1}= & 12 \sqrt{k^{2}+\omega^{2}}, \theta=-\frac{\sqrt{k^{2}+\omega^{2}}}{k \omega}, q=k\left(x+\frac{1}{\Gamma(\mu)}\right)^{\mu} \\
& -\omega\left(t+\frac{1}{\Gamma(\mu)}\right)^{\mu} .
\end{aligned}
$$




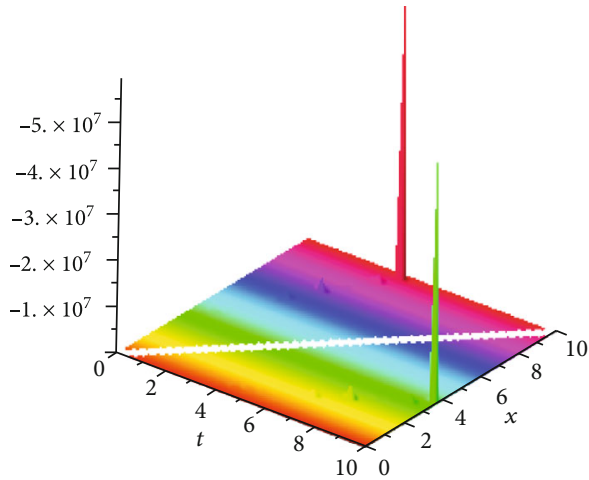

(a)

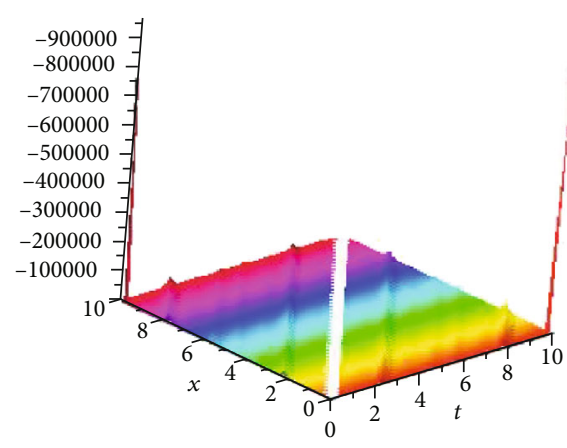

(b)

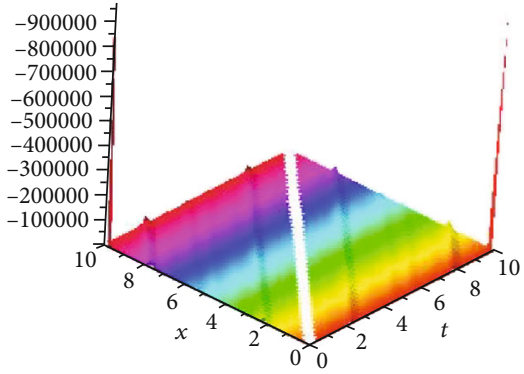

(c)

FIGURE 6: The different types of graphs to periodic solution (117) for the parameters $\delta=-2, d=1, k=2$, and $\omega=2$ with providing amounts of (a) $\mu=0.5$, (b) $\mu=0.75$, and (c) $\mu=1$ within the interval $0 \leq x, t \leq 10$.

Sixth:

$$
\begin{aligned}
\delta= & -\frac{144 k^{2}+144 \omega^{2}-\tau_{1}^{2}}{576 k^{2} \omega^{2}}, \theta=-\frac{1}{12} \frac{\tau_{1}}{k \omega}, \pi_{0} \\
= & \frac{1}{48} \frac{48 k^{2}+48 \omega^{2}-\tau_{1}^{2}}{k \omega}, \tau_{2}=-12 k \omega .
\end{aligned}
$$

According to Family II, (112) can be written as

$$
\begin{aligned}
\Psi_{9}(q)= & -\frac{1}{48} \frac{48 k^{2}+48 \omega^{2}-+\tau_{1}^{2}}{k \omega} \\
& +\tau_{1}\left(\frac{\sqrt{-\theta^{2}+4 \delta}}{2 \delta} \tan \left(\frac{\sqrt{-\theta^{2}+4 \delta}}{2}(q+\Sigma)\right)-\frac{\theta}{2 \delta}\right) \\
& -12 k \omega\left(\frac{\sqrt{-\theta^{2}+4 \delta}}{2 \delta} \tan \left(\frac{\sqrt{-\theta^{2}+4 \delta}}{2}(q+\Sigma .)\right)-\frac{\theta}{2 \delta}\right)^{2},
\end{aligned}
$$

where

$$
\begin{aligned}
\frac{\theta}{2 \delta}= & -\frac{24 \tau_{1} k \omega}{144 k^{2}+144 \omega^{2}+\tau_{1}^{2}}, \theta^{2}-4 \delta=-\frac{k^{2}+\omega^{2}}{k^{2} \omega^{2}} \\
& <0, q=k\left(x+\frac{1}{\Gamma(\mu)}\right)^{\mu}-\omega\left(t+\frac{1}{\Gamma(\mu)}\right)^{\mu} .
\end{aligned}
$$

\section{Results and Discussion}

In this section, we will discuss the physical explanation of the found exact solutions to three nonlinear fractional models. We expose the graphical representation of these solutions and accomplish the different kinds of solution. To sketch plots, we have utilized the Maple software package. Every exact solutions are offered in a Maple 3D plot view for proper understanding. Some appropriate values are given in Figures 1-6 to analyze the dynamic properties briefly. These figures are dependent on the family conditions which are of importance physically. It has been investigated that all figures include a $3 \mathrm{D}$ plot with three fractional-order $\mu=0.5, \mu=0.75$ , and $\mu=1$ designed for three nonlinear fractional models involving the $\mu$-derivative.

\section{Conclusion}

In this study, the efficient and significant solutions to three nonlinear fractional models were established which include kink solution, periodic wave solution, singular kink, single soliton, and other types of soliton found by preferring different free parameters. These applied parameters have important conjugation, such as choosing various inputs of free parameters from an individual solution; known solutions must be found identically. It is important to notice that the new type solution of the space-time fractional $(1+1)$-dimensional Boussinesq equation, $(2+1)$ -dimensional breaking soliton equations, and SRLW equations has not been exposed by the oncoming $\exp (-\Omega(q))$ -expansion technique in the previous literature. So we claim that in this current study, the obtained solutions are unique and thus could be more effective in the study of space-time fractional nonlinear physical phenomena. All calculations in this paper have been made quickly with the aid of Maple. The development of offered methods may allow the mitigating Internet bottleneck with quadratic-cubic nonlinearity to be used in more general configurations. The solutions are all verified by putting them back into the original equations with the aid of the Maple symbolic computation package 18 .

\section{Data Availability}

The datasets supporting the conclusions of this article are included in the article.

\section{Conflicts of Interest}

The authors declare that they have no conflicts of interest.

\section{Authors' Contributions}

The authors made contribution to this work. QL made the numerical simulations and wrote some sections of the article. DS, OAI, GS, and JM provided the remaining sections. JM and DS provided the conclusions. QL and OAI provided Section 4. GS and JM wrote Section 5. Also, QL, 
DS, and JM provided figures. Moreover, JM and OAI provided the references. The authors read and approved the final manuscript.

\section{References}

[1] K. S. Miller and B. Ross, An Introduction to the Fractional Calculus and Fractional Differential Equations, Wiley, 1993.

[2] I. Podlubny, Fractional Differential Equations, 198 Academic Press, San Diego, CA, USA, 1999.

[3] I. Karahan and M. Ozdemir, "A new iterative projection method for approximating fixed point problems and variational inequality problems," Proceedings of the Institute of Mathematics and Mechanics, vol. 40, pp. 68-79, 2014.

[4] Y. Y. Mammadov, "Weighted inequalities for dunkl fractional maximal function and dunkl fractional integrals," Proceedings of the Institute of Mathematics and Mechanics, vol. 40, pp. 93103, 2014.

[5] E. H. Khalilov, "On approximate solution of a singular integral equation of Neumann's external boundary value problem for a wave equation," Proceedings of the Institute of Mathematics and Mechanics, vol. 41, pp. 91-105, 2015.

[6] E. S. H. Mamedov, "Variational principle for two-parameter spectral problem under left definiteness condition," Proceedings of the Institute of Mathematics and Mechanics, vol. 41, pp. 124-129, 2015.

[7] I. Aziz and Q. Ul Ain, "Numerical solution of partial integrodifferential equations with weakly singular kernels," Advanced Mathematical Models \& Applications, vol. 5, no. 2, pp. 149$160,2020$.

[8] R. M. Ganji and H. Jafari, "Numerical solution of variable order integro-differential equations," Advanced Mathematical Models \& Applications, vol. 4, no. 1, pp. 64-69, 2019.

[9] S. S. Yusubov, "Boundary value problems for hyperbolic equations with a Caputo fractional derivative," Advanced Mathematical Models \& Applications, vol. 5, no. 2, pp. 192-204, 2020.

[10] J. Manafian and S. Heidari, "Periodic and singular kink solutions of the Hamiltonian amplitude equation," Advanced Mathematical Models \& Applications, vol. 4, no. 2, pp. 134149, 2019.

[11] A. A. Kilbas, H. M. Srivastava, and J. J. Trujillo, Theory and Applications of Fractional Differential Equations, Elsevier, Amsterdam, 2006.

[12] H. C. Yaslan, "Legendre collocation method for the nonlinear space-time fractional partial differential equations," Iranian Journal of Science and Technology, Transactions A: Science, vol. 44, no. 1, pp. 239-249, 2020.

[13] B. Zheng, "( $\left.G^{\prime} / G\right)$-expansion method for solving fractional partial differential equations in the theory of mathematical physics," Communications in Theoretical Physics, vol. 58, no. 5, pp. 623-630, 2012.

[14] A. C. Gómez and A. H. Salas, "The variational iteration method combined with improved generalized tanh-coth method applied to Sawada-Kotera equation," Applied Mathematics and Computation, vol. 217, no. 4, pp. 1408-1414, 2010.

[15] S. Zhang and H. Q. Zhang, "Fractional sub-equation method and its applications to nonlinear fractional PDEs," Physics Letters A, vol. 375, no. 7, pp. 1069-1073, 2011.

[16] A. Houwe, M. Inc, S. Y. Doka, M. A. Akinlar, and D. Baleanu, "Chirped solitons in negative index materials generated by
Kerr nonlinearity," Results in Physics, vol. 17, article 103097, 2020.

[17] M. H. Bashar and M. M. Roshid, "Rouge wave solutions of a nonlinear pseudo-parabolic physical model through the advance exponential expansion method," International Journal of Physical Research, vol. 8, no. 1, pp. 1-7, 2020.

[18] I. Simbanefayi and C. M. Khalique, "Travelling wave solutions and conservation laws for the Korteweg-de Vries- BejaminBona-Mahony equation," Results in Physics, vol. 8, pp. 57-63, 2018.

[19] J. Manafian, B. Mohammadi Ivatlo, and M. Abapour, "Lumptype solutions and interaction phenomenon to the $(2+1)$ dimensional breaking Soliton equation," Applied Mathematics and Computation, vol. 13, pp. 13-41, 2019.

[20] O. A. Ilhan and J. Manafian, "Periodic type and periodic crosskink wave solutions to the $(2+1)$-dimensional breaking soliton equation arising in fluid dynamics," Modern Physics Letters B, vol. 33, no. 23, article 1950277, 2019.

[21] H. Ali, M. Shahadat, M. M. Miah, and M. A. Akbar, "Study of abundant explicit wave solutions of the Drinfeld-Sokolov-Satsuma-Hirota (DSSH) equation and the shallow water wave equation," Propulsion and Power Research, vol. 7, no. 4, pp. 320-328, 2018.

[22] O. A. Ilhan, J. Manafian, A. Alizadeh, and H. M. Baskonus, "New exact solutions for nematicons in liquid crystals by the $\tan (\phi / 2) \$ \$$-expansion method arising in fluid mechanics," The European Physical Journal Plus, vol. 135, no. 3, p. 313, 2020.

[23] J. Manafian, S. A. Mohammed, A. Alizadeh, H. M. Baskonus, and W. Gao, "Investigating lump and its interaction for the third-order evolution equation arising propagation of long waves over shallow water," European Journal of Mechanics /B Fluids, vol. 84, pp. 289-301, 2020.

[24] K. S. Nisar, O. A. Ilhan, J. Manafian, M. Shahriari, and D. Soybaş, "Analytical behavior of the fractional Bogoyavlenskii equations with conformable derivative using two distinct reliable methods," Results in Physics, vol. 22, article 103975, 2021.

[25] M. M. Rahhman, A. Aktar, and K. C. Roy, “Analytical solutions of nonlinear coupled Schrodinger KdV equation via advance exponential expansion," American Journal of Mathematical and Computer Modelling, vol. 3, no. 3, pp. 46-51, 2018.

[26] H. Jafari, H. Tajadodi, D. Baleanu, A. A. Al-Zahrani, Y. A. Alhamed, and A. H. Zahid, "Exact solutions of Boussinesq and $\mathrm{KdV}-\mathrm{mKdV}$ equations by fractional sub-equation method," Romanian Reports in Physics, vol. 65, no. 4, pp. 1119-1124, 2013.

[27] S. T. Mohyud-Din and S. Bibi, "Exact solutions for nonlinear fractional differential equations using exponential rational function method," Optical Quantum and Electron, vol. 49, no. 2, p. 64, 2017.

[28] F. Xu, Y. Gao, and W. Zhang, "Construction of analytic solution for time-fractional Boussinesq equation using iterative method," Advances in Mathematical Physics, vol. 2015, Article ID 506140, 7 pages, 2015.

[29] A. A. Hemeda, "Solution of fractional partial differential equations in fluid mechanics by extension of some iterative method," Abstract and Applied Analysis, vol. 2013, Article ID 717540, 9 pages, 2013.

[30] M. P. Haghighi and J. Manafian, "Solving a class of boundary value problems and fractional Boussinesq-like equation with 
$\beta$-derivatives by fractional-order exponential trial functions," Journal of Ocean Engineering and Science, vol. 5, no. 3, pp. 197-204, 2020.

[31] C. Wen and B. Zheng, "A new fractional sub-equation method for fractional partial differential equations," WSEAS Transactions on Mathematics, vol. 12, pp. 564-571, 2013.

[32] F. Meng and Q. Feng, "Exact solutions with variable coefficient function forms for conformable fractional partial differential equations by an auxiliary equation method," Advances in Mathematical Physics, vol. 2018, Article ID 4596506, 8 pages, 2018.

[33] F. Xu, "Application of Exp-function method to symmetric regularized long wave (SRLW) equation," Physics Letters A, vol. 372, no. 3, pp. 252-257, 2008.

[34] J. F. Alzaidy, "The fractional sub-equation method and exact analytical solutions for some nonlinear fractional PDEs," American Journal of Mathematical Analysis, vol. 1, no. 1, pp. 14-19, 2013.

[35] Ö. Güner and D. Eser, "Exact solutions of the space time fractional symmetric regularized long wave equation using different methods," Advances in Mathematical Physics, vol. 2014, Article ID 456804, 8 pages, 2014.

[36] A. Ataganda, "Fractional discretization: the African's tortoise walk," Chaos, Solitons \& Fractal, vol. 130, article 109399, 2020.

[37] A. Ataganda, "Blind in a commutative world: simple illustrations with functions and chaotic attractors," Chaos, Solitons \& Fractal, vol. 114, pp. 347-363, 2018.

[38] A. Ataganda, "Fractal-fractional differentiation and integration: connecting fractal calculus and fractional calculus to predict complex system," Chaos, Solitons \& Fractal, vol. 114, pp. 396-406, 2017.

[39] A. R. Seadawy and J. Manafian, "New soliton solution to the longitudinal wave equation in a magneto-electro-elastic circular rod," Results Physics, vol. 8, pp. 1158-1167, 2018.

[40] A. R. Seadawy and S. Z. Alamri, "Mathematical methods via the nonlinear two-dimensional water waves of Olver dynamical equation and its exact solitary wave solutions," Results in Physics, vol. 8, pp. 286-291, 2018.

[41] M. Iqbal, A. R. Seadawy, O. H. Khalil, and D. Lu, "Propagation of long internal waves in density stratified ocean for the (2+1)dimensional nonlinear Nizhnik-Novikov-Vesselov dynamical equation," Results in Physics, vol. 16, article 102838, 2020.

[42] Abdullah, A. R. Seadawy, and W. Jun, "Mathematical methods and solitary wave solutions of three-dimensional ZakharovKuznetsov-Burgers equation in dusty plasma and its applications," Results in Physics, vol. 7, pp. 4269-4277, 2017.

[43] A. R. Seadawy and K. el-Rashidy, "Dispersive solitary wave solutions of Kadomtsev-Petviashvili and modified Kadomtsev-Petviashvili dynamical equations in unmagnetized dust plasma," Results in Physics, vol. 8, pp. 1216-1222, 2018.

[44] M. Iqbal, A. R. Seadawy, and D. Lu, "Construction of solitary wave solutions to the nonlinear modified Kortewege-de Vries dynamical equation in unmagnetized plasma via mathematical methods," Modern Physics Lettters B, vol. 33, article 1850183, pp. 1-13, 2018.

[45] N. Cheemaa, S. Chen, and A. R. Seadawy, "Propagation of isolated waves of coupled nonlinear $(2+1)$-dimensional Maccari system in plasma physics," Results in Physics, vol. 17, article 102987, 2020.

[46] N. Nasreen, A. R. Seadawy, D. Lu, and M. Arshad, "Construction of modulation instability analysis and optical soliton solu- tions of pertubed nonlinear Schrodinger dynamical equation with power law nonlinearity in non-kerr medium," Results in Physics, vol. 13, article 102263, 2019.

[47] H. Ahmad, A. R. Seadawy, T. A. Khan, and P. Thounthong, "Analytic approximate solutions for some nonlinear Parabolic dynamical wave equations," Taibah University Journal of Science, vol. 14, no. 1, pp. 346-358, 2020.

[48] E. S. Selima, A. R. Seadawy, and X. Yao, “The nonlinear dispersive Davey-Stewartson system for surface waves propagation in shallow water and its stability," The European Physical Journal Plus, vol. 131, no. 12, p. 425, 2016.

[49] S. Saha Ray, "Dispersive optical solitons of time-fractional Schrödinger-Hirota equation in nonlinear optical fibers," Physica A: Statistical Mechanics and its Applications, vol. 537, article 122619, 2020.

[50] S. Sahoo and S. Saha Ray, "The new soliton wave solutions of conformable time-fractional Rosenau-Kawahara-RLW equation," Modern Physics Letters B, vol. 33, no. 29, article 1950365, 2019.

[51] M. Inc, A. Yusuf, A. I. Aliyu, and D. Baleanu, "Soliton structures to some time-fractional nonlinear differential equations with conformable derivative," Optical and Quantum Electronic, vol. 50, no. 1, p. 20, 2018.

[52] S. Salahshour, A. Ahmadian, S. Abbasbandy, and D. Baleanu, "M-fractional derivative under interval uncertainty: theory, properties and applications," Chaos, Solitons \& Fractal, vol. 117, pp. 84-93, 2018.

[53] M. Inc, A. I. Aliyu, A. Yusuf, D. Baleanu, and E. Nuray, "Complexiton and solitary wave solutions of the coupled nonlinear Maccari's system using two integration schemes," Modern Physics Letters B, vol. 32, no. 2, article 1850014, 2018.

[54] A. T. Ali and E. R. Hassan, "General Expa-function method for nonlinear evolution equations," Applied Mathematics and Computation, vol. 217, no. 2, pp. 451-459, 2010.

[55] M. Abdelrahman, E. H. M. Zahran, and M. M. A. Khater, "The $\exp -(-\phi(\xi))$-expansion method and its application for solving nonlinear evolution equations," International Journal of Modern Nonlinear Theory and Application, vol. 4, no. 1, pp. 37-47, 2015.

[56] S. M. R. Islam, K. Khan, and M. A. Akbar, "Study of exp- $(-\phi(\xi)$ )-expansion method for solving nonlinear partial differential equations," British Journal of Mathematics \& Computer Science, vol. 5, pp. 397-407, 2014.

[57] K. Khan and M. A. Akbar, "The exp- $(-\phi(\xi))$-expansion method for finding travelling wave solutions of VakhnenkoParkes equation," International Journal of Dynamical Systems and Differential Equations, vol. 5, no. 1, pp. 72-83, 2014.

[58] K. Nematollah, F. Michal, and K. Yasser, "Application of the $\exp (-\varphi)$-expansion method to the Pochhammer-Chree equation," Univerzitet u Nišu, vol. 32, pp. 3347-3354, 2018.

[59] J. Manafian, “Optical soliton solutions for Schrödinger type nonlinear evolution equations by the $\tan (\Phi(\xi) / 2)$-expansion method," Optik, vol. 127, no. 10, pp. 4222-4245, 2016.

[60] A. Atangana and D. Baleanu, "New fractional derivatives with nonlocal and non-singular kernel. Theory and application to heat transfer model," Thermal Science, vol. 20, no. 2, pp. 763-769, 2016. 